This is a self-archived version of an original article. This version may differ from the original in pagination and typographic details.

Author(s): Parkkonen, Jouni; Paulin, Frédéric

Title: On the nonarchimedean quadratic Lagrange spectra

Year: 2020

Version: Accepted version (Final draft)

Copyright: @ C Springer-Verlag GmbH Germany, part of Springer Nature 2019

Rights: In Copyright

Rights url: http://rightsstatements.org/page/lnC/1.0/?language=en

Please cite the original version:

Parkkonen, J., \& Paulin, F. (2020). On the nonarchimedean quadratic Lagrange spectra.

Mathematische Zeitschrift, 294(3-4), 1065-1084. https://doi.org/10.1007/s00209-019-02300-1 


\title{
On the nonarchimedean quadratic Lagrange spectra
}

\author{
Jouni Parkkonen Frédéric Paulin
}

March 8, 2019

\begin{abstract}
We study Diophantine approximation in completions of functions fields over finite fields, and in particular in fields of formal Laurent series over finite fields. We introduce a Lagrange spectrum for the approximation by orbits of quadratic irrationals under the modular group. We give nonarchimedean analogs of various well known results in the real case: the closedness and boundedness of the Lagrange spectrum, the existence of a Hall ray, as well as computations of various Hurwitz constants. We use geometric methods of group actions on Bruhat-Tits trees. ${ }^{1}$
\end{abstract}

\section{Introduction}

Diophantine approximation in local fields of positive characteristic has been studied for many years, both from the classical viewpoint (see the works of de Mathan, Lasjaunias, Osgood, W. Schmidt, Thakur, Voloch for instance) and from the point of view of arithmetic geometry (see the works of Mahler, Manin, and many others), as well from an ergodic theory viewpoint (see for instance $[\mathrm{BeN}]$ ). In this paper, we are interested in the approximation by quadratic irrationals: we define and study the quadratic Lagrange spectra in completions of function fields over finite fields with respect to the absolute values defined by discrete valuations. In this introduction, we concentrate on the special case of the field of rational fractions and its valuation at infinity. We refer to Section 3 for the definitions and results in the general case, allowing congruence considerations.

Let $\mathbb{F}_{q}$ be a finite field of order a positive power $q$ of a positive prime. Let $R=\mathbb{F}_{q}[Y]$, $K=\mathbb{F}_{q}(Y)$ and $\widehat{K}=\mathbb{F}_{q}\left(\left(Y^{-1}\right)\right)$ be respectively the ring of polynomials in one variable $Y$ over $\mathbb{F}_{q}$, the field of rational functions in $Y$ over $\mathbb{F}_{q}$ and the field of formal Laurent series in $Y^{-1}$ over $\mathbb{F}_{q}$. Then $\widehat{K}$ is a nonarchimedean local field, the completion of $K$ with respect to its place at infinity, that is, the absolute value $\left|\frac{P}{Q}\right|=q^{\operatorname{deg} P-\operatorname{deg} Q}$ for all $P, Q \in R-\{0\}$. Let

$$
\mathrm{QI}=\{f \in \widehat{K}:[K(f): K]=2\}
$$

be the set of quadratic irrationals over $K$ in $\widehat{K}$. Given $f \in \widehat{K}-K$, it is well known that $f \in \mathrm{QI}$ if and only if the continued fraction expansion ${ }^{2}$ of $f$ is eventually periodic. The projective action of $\Gamma=\mathrm{PGL}_{2}(R)$ on $\mathbb{P}_{1}(\widehat{K})=\widehat{K} \cup\{\infty\}$ preserves QI, keeping the periodic part of the continued fraction expansions unchanged (up to cyclic permutation

\footnotetext{
${ }^{1}$ Keywords: quadratic irrational, continued fraction expansion, positive characteristic, formal Laurent series, Lagrange spectrum, Hurwitz constant, Hall ray. AMS codes: 11J06, 11J70, 11R11, 20E08, 20G25

${ }^{2}$ See Section 4 for a definition.
} 
and invertible elements). We refer for instance to [Las, Sch, Pau] for background on the above notions.

Now let us fix $\alpha \in$ QI. We denote by $\alpha^{\sigma} \in$ QI the Galois conjugate of $\alpha$ over $K$. The complexity $h(\alpha)=\frac{1}{\left|\alpha-\alpha^{\sigma}\right|}$ of $\alpha$ was introduced in [HP] and developped in [BPP, §17.2]. It plays the role of the (naive) height of a rational number in Diophantine approximation by rationals, and is an appropriate complexity when studying the approximation by elements in the orbit under the modular group of a given quadratic irrational. ${ }^{3}$ We refer to the above references for motivations and results, in particular to [HP, Thm. 1.6] for a Khintchine type result and to $[\mathrm{BPP}, \S 17.2]$ for an equidistribution result of the orbit of $\alpha$ under $\mathrm{PGL}_{2}(R)$.

Let

$$
\Theta_{\alpha}=\mathrm{PGL}_{2}(R) \cdot\left\{\alpha, \alpha^{\sigma}\right\}
$$

be the union of the orbits of $\alpha$ and $\alpha^{\sigma}$ under the projective action of $\mathrm{PGL}_{2}(R)$. Given $x \in \widehat{K}-\left(K \cup \Theta_{\alpha}\right)$, we define the quadratic approximation constant of $x$ by

$$
c_{\alpha}(x)=\liminf _{\beta \in \Theta_{\alpha},\left|\beta-\beta^{\sigma}\right| \rightarrow 0} \frac{|x-\beta|}{\left|\beta-\beta^{\sigma}\right|} .
$$

We define the quadratic Lagrange spectrum of $\alpha$ as

$$
\operatorname{Sp}(\alpha)=\left\{c_{\alpha}(x): x \in \widehat{K}-\left(K \cup \Theta_{\alpha}\right)\right\} .
$$

Note that $\operatorname{Sp}(\alpha) \subset q^{\mathbb{Z}} \cup\{0,+\infty\}$. It follows from [HP, Thm. 1.6] that if $m_{\widehat{K}}$ is a Haar measure on the locally compact additive group of $\widehat{K}$, then for $m_{\widehat{K}}$-almost every $x \in \widehat{K}$, we have $c_{\alpha}(x)=0$. Hence in particular, $0 \in \operatorname{Sp}(\alpha)$ and the quadratic Lagrange spectrum is therefore closed. In Section 3, we prove that it is bounded, and we can thus define the (quadratic) Hurwitz constant of $\alpha$ as $\max \operatorname{Sp}(\alpha) \in q^{\mathbb{Z}}$.

The following theorems, giving nonarchimedean analogs of the results of Lin, Bugeaud and Pejkovic [Lin, Bug1, Pej], say that the quadratic Lagrange spectrum of $\alpha$ is a closed bounded subset of $q^{\mathbb{Z}} \cup\{0\}$ which contains an initial interval, and computes various Hurwitz constants.

Theorem 1.1. Let $\alpha$ be a quadratic irrational over $K$ in $\widehat{K}$.

(1) (Upper bound) Its quadratic Hurwitz constant satisfies $\max \operatorname{Sp}(\alpha) \leq q^{-2}$.

(2) (Hall ray) There exists $m_{\alpha} \in \mathbb{N}$ such that for all $n \in \mathbb{N}$ with $n \geq m_{\alpha}$, we have $q^{-n} \in \operatorname{Sp}(\alpha)$.

In Section 3, we even prove that Assertion (2) of this theorem is valid when $K$ is any function field over $\mathbb{F}_{q}, \widehat{K}$ is the completion of $K$ at any place of $K$, and $R$ is the corresponding affine function ring.

Theorem 1.2. The Hurwitz constant of any quadratic irrational over $K$ in $\widehat{K}$, whose continued fraction expansion is eventually $k$-periodic with $k \leq q-1$, is equal to $q^{-2}$.

There are examples of quadratic irrationals for which the quadratic Lagrange spectrum coincides with the maximal Hall ray. The following theorem gives a special case, see Theorem 4.11 for a more general result.

\footnotetext{
${ }^{3}$ see [PaP4] for a comparison between the naive height of the algebraic number $\alpha$ and the above complexity of $\alpha$ in the Archimedean case.
} 
Theorem 1.3. If

$$
\varphi=Y+\frac{1}{Y+\frac{1}{Y+\cdots}},
$$

then $\operatorname{Sp}(\varphi)=\{0\} \cup\left\{q^{-n-2}: n \in \mathbb{N}\right\}$.

In Proposition 4.12, we give a class of quadratic irrationals whose quadratic Lagrange spectrum does not coincide with its maximal Hall ray, in other words, who have gaps in their spectrum.

After the first version of this paper was posted on ArXiv, Yann Bugeaud [Bug2] has given a completely different proof of the above results (except the generalisation to function fields), and proved several new theorems giving a more precise description of these spectra. In particular, he proved that all approximation constants for a given quadratic irrational are attained on the other quadratic irrationals, that for every $m \geq 2$ there exists $\beta \in \mathrm{QI}$ such that $\max \operatorname{Sp}(\beta)=q^{-m}$, and that for all $\ell \in \mathbb{N}$, there exists $\beta \in$ QI such that $\operatorname{Sp}(\beta)$ contains exactly $\ell$ gaps.

In order to explain the origin of our results, recall that for $x \in \mathbb{R}-\mathbb{Q}$, the approximation constant of $x$ by rational numbers is

$$
c(x)=\liminf _{p, q \in \mathbb{Z}, q \rightarrow+\infty} q^{2}\left|x-\frac{p}{q}\right|,
$$

and that the Lagrange spectrum is $\operatorname{Sp}_{\mathbb{Q}}=\{c(x): x \in \mathbb{R}-\mathbb{Q}\}$. Numerous properties of the Lagrange spectrum are known, see for instance $[\mathrm{CF}]$. In particular, $\mathrm{Sp}_{\mathbb{Q}}$ is bounded and closed, has maximum $\frac{1}{\sqrt{5}}$, and contains a maximal interval $[0, \mu]$ with $0<\mu<\frac{1}{\sqrt{5}}$ called a Hall ray. Khinchin [Khi] proved that almost every real number is badly approximable by rational numbers, so that the approximation constant vanishes almost surely. Many of these results have been generalised to the Diophantine approximation of complex numbers, Hamiltonian quaternions and for the Heisenberg group, see for example [Poi, Sch1, Sch2, PaP1, PaP2, PaP3].

Let $\alpha_{0}$ be a fixed real quadratic irrational number over $\mathbb{Q}$. For every such number $\alpha$, let $\alpha^{\sigma}$ be its Galois conjugate. Let $\mathscr{E}_{\alpha_{0}}=\mathrm{PSL}_{2}(\mathbb{Z}) \cdot\left\{\alpha_{0}, \alpha_{0}^{\sigma}\right\}$ be its (countable, dense in $\mathbb{R}$ ) orbit for the action by homographies and anti-homographies of $\mathrm{PSL}_{2}(\mathbb{Z})$ on $\mathbb{R} \cup\{\infty\}$. For every $x \in \mathbb{R}-\left(\mathbb{Q} \cup \mathscr{E}_{\alpha_{0}}\right)$, the approximation constant of $x$ by elements of $\mathscr{E}_{\alpha_{0}}$ was defined in $[\mathrm{PaP} 4]$ by

$$
c_{\alpha_{0}}(x)=\liminf _{\alpha \in \mathscr{E}_{\alpha_{0}}:\left|\alpha-\alpha^{\sigma}\right| \rightarrow 0} 2 \frac{|x-\alpha|}{\left|\alpha-\alpha^{\sigma}\right|},
$$

the quadratic Lagrange spectrum (or approximation spectrum) of $\alpha_{0}$ by

$$
\operatorname{Sp}\left(\alpha_{0}\right)=\left\{c_{\alpha_{0}}(x): x \in \mathbb{R}-\left(\mathbb{Q} \cup \mathscr{E}_{\alpha_{0}}\right)\right\}
$$

and the Hurwitz constant of $\alpha_{0}$ by $\sup \operatorname{Sp}\left(\alpha_{0}\right)$. We proved that the quadratic Lagrange spectrum of $\alpha_{0}$ is bounded and closed, and that an analog of Khinchin's theorem holds. We generalised the definitions and the above results to the approximation of complex numbers and elements of the Heisenberg group. In the latter cases, we also proved the existence of a Hall ray in the spectrum. 
In the real case, the existence of a Hall ray in $\operatorname{Sp}\left(\alpha_{0}\right)$ is due to Lin [Lin]. Bugeaud [Bug1] proved that the Hurwitz constant of the Golden Ratio $\phi$ is equal to $\frac{3}{\sqrt{5}}-1$, and his conjecture that the Hurwitz constant of any real quadratic irrational is at most $\frac{3}{\sqrt{5}}-1$ was confirmed by Pejkovic [Pej]. The Hurwitz constant is known explicitly in many 2-periodic continued fraction expansion cases, see [Pej, Lin].

Acknowledgements: This work was supported by the French-Finnish CNRS grant PICS №6950. We thank a lot Yann Bugeaud for his comments on the first version of this paper, which allowed us to remove the unnecessary assumption of odd characteristic, and gave a negative solution to a conjecture we proposed on a general formula for the Hurwitz constants.

\section{Background on function fields and Bruhat-Tits trees}

In this section, we recall the basic notations and properties of function fields $K$ over $\mathbb{F}_{q}$ and their valuations $v$, the associated Bruhat-Tits trees $\mathbb{T}_{v}$ and modular groups $\Gamma_{v}$ acting on $\mathbb{T}_{v}$. We refer to [Gos, Ros, Ser] for definitions, proofs and further information, see also [BPP, Ch. 14 and 15].

Let $\mathbb{F}_{q}$ be a finite field of order $q$ with $q$ a positive power of a positive prime.

\section{$2.1 \quad$ Function fields}

Let $K$ be a function field over $\mathbb{F}_{q}$ and let $v: K^{\times} \rightarrow \mathbb{Z}$ be a (normalised discrete) valuation of $K$. Let $R_{v}$ be the affine function ring associated with $(K, v)$. Let $|\cdot|_{v}$ be the absolute value on $K$ corresponding to $v$ and let $K_{v}$ be the completion of $K$ with respect to $|\cdot|_{v}$. We again denote by $v$ and $|\cdot|_{v}$ the extensions of $v$ and $|\cdot|_{v}$ to $K_{v}$. Let

$$
\mathscr{O}_{v}=\left\{x \in K_{v}: v(x) \geq 0\right\}
$$

be the valuation ring of $K_{v}$. Its unique maximal ideal is

$$
\mathfrak{m}_{v}=\left\{x \in K_{v}: v(x)>0\right\} .
$$

We denote the cardinality of the residual field $k_{v}=\mathscr{O}_{v} / \mathfrak{m}_{v}$ by $q_{v}$, so that $|f|_{v}=q_{v}^{-v(f)}$ for all $f \in K_{v}^{\times}$.

Example 2.1. Let $K=\mathbb{F}_{q}(Y)$ be the field of rational functions in one variable $Y$ with coefficients in $\mathbb{F}_{q}$, let $\mathbb{F}_{q}[Y]$ be the ring of polynomials in one variable $Y$ with coefficients in $\mathbb{F}_{q}$, and let $v_{\infty}: K^{\times} \rightarrow \mathbb{Z}$ be the valuation at infinity of $K$, defined on every $P / Q \in K$ with $P, Q \in \mathbb{F}_{q}[Y]-\{0\}$ by

$$
v_{\infty}(P / Q)=\operatorname{deg} Q-\operatorname{deg} P
$$

Then $R_{v_{\infty}}=\mathbb{F}_{q}[Y]$ and the absolute value $|\cdot|_{v_{\infty}}$ on $K$ associated with $v_{\infty}$ is the absolute value defined in the introduction. The completion $K_{v_{\infty}}$ of $K$ for $|\cdot|_{v_{\infty}}$ is the field $\mathbb{F}_{q}\left(\left(Y^{-1}\right)\right)$ of formal Laurent series in one variable $Y^{-1}$ with coefficients in $\mathbb{F}_{q}$, denoted by $\widehat{K}$ in the introduction. The elements $x$ in $\mathbb{F}_{q}\left(\left(Y^{-1}\right)\right)$ are of the form

$$
x=\sum_{i \in \mathbb{Z}} x_{i} Y^{-i}
$$


where $x_{i} \in \mathbb{F}_{q}$ for all $i \in \mathbb{Z}$ and $x_{i}=0$ for $i$ small enough. The valuation at infinity of $\mathbb{F}_{q}\left(\left(Y^{-1}\right)\right)$ extending the valuation at infinity of $\mathbb{F}_{q}(Y)$ is

$$
\forall x \in \mathbb{F}_{q}\left(\left(Y^{-1}\right)\right)^{\times}, \quad v_{\infty}(x)=\sup \left\{i \in \mathbb{Z}: \forall j<i, \quad x_{j}=0\right\} .
$$

The valuation ring $\mathscr{O}_{v_{\infty}}$ of $v_{\infty}$ is the ring $\mathbb{F}_{q}\left[\left[Y^{-1}\right]\right]$ of formal power series in one variable $Y^{-1}$ with coefficients in $\mathbb{F}_{q}$. The residual field $k_{v_{\infty}}=\mathscr{O}_{v_{\infty}} / \mathfrak{m}_{v_{\infty}}$ of $v_{\infty}$ is $\mathbb{F}_{q}$, which has order $q_{v_{\infty}}=q$.

We identify the projective line $\mathbb{P}_{1}\left(K_{v}\right)$ with $K_{v} \cup\{\infty\}$ using the map $K_{v}(x, y) \mapsto \frac{x}{y}$, so that

$$
\infty=[1: 0] .
$$

The projective action of $\mathrm{PGL}_{2}\left(K_{v}\right)$ on $\mathbb{P}^{1}\left(K_{v}\right)$ is the action by homographies on $K_{v} \cup\{\infty\}$, given by $(g, z) \mapsto g \cdot z=\frac{a z+b}{c z+d}$ if $g=\left[\begin{array}{ll}a & b \\ c & d\end{array}\right] \in \operatorname{PGL}_{2}\left(K_{v}\right)$. As usual, we define $g \cdot \infty=\frac{a}{c}$ and $g \cdot\left(-\frac{d}{c}\right)=\infty$.

\subsection{Bruhat-Tits trees}

An $\mathscr{O}_{v}$-lattice $\Lambda$ in the $K_{v}$-vector space $K_{v} \times K_{v}$ is a rank 2 free $\mathscr{O}_{v}$-submodule of $K_{v} \times K_{v}$, generating $K_{v} \times K_{v}$ as a vector space. The Bruhat-Tits tree $\mathbb{T}_{v}$ of $\left(\mathrm{PGL}_{2}, K_{v}\right)$ is the graph whose set of vertices $V \mathbb{T}_{v}$ is the set of homothety classes (under $\left.\left(K_{v}\right)^{\times}\right)[\Lambda]$ of $\mathscr{O}_{v}$-lattices $\Lambda$ in $K_{v} \times K_{v}$, and whose set of edges $E \mathbb{T}_{v}$ is the set of pairs $\left(x, x^{\prime}\right)$ of vertices such that there exist representatives $\Lambda$ of $x$ and $\Lambda^{\prime}$ of $x^{\prime}$ for which $\Lambda \subset \Lambda^{\prime}$ and $\Lambda^{\prime} / \Lambda$ is isomorphic to $\mathscr{O}_{v} / \mathfrak{m}_{v}$. The graph $\mathbb{T}_{v}$ is a regular tree of degree $\left|\mathbb{P}_{1}\left(k_{v}\right)\right|=q_{v}+1$. The standard base point $*_{v}$ of $\mathbb{T}_{v}$ is the homothety class $\left[\mathscr{O}_{v} \times \mathscr{O}_{v}\right]$ of the $\mathscr{O}_{v}$-lattice $\mathscr{O}_{v} \times \mathscr{O}_{v}$ generated by the canonical basis of $K_{v} \times K_{v}$. The link

$$
\operatorname{lk}\left(*_{v}\right)=\left\{y \in V \mathbb{T}_{v}: d\left(y, *_{v}\right)=1\right\}
$$

of $*_{v}$ in $\mathbb{T}_{v}$ identifies with the projective line $\mathbb{P}_{1}\left(k_{v}\right)$.

The left linear action of $\mathrm{GL}_{2}\left(K_{v}\right)$ on $K_{v} \times K_{v}$ induces a faithful, vertex-transitive left action of $\mathrm{PGL}_{2}\left(K_{v}\right)$ by automorphisms on $\mathbb{T}_{v}$. The stabiliser of $*_{v}$ in $\mathrm{PGL}_{2}\left(K_{v}\right)$ is $\mathrm{PGL}_{2}\left(\mathscr{O}_{v}\right)$, which acts projectively on $\operatorname{lk}\left(*_{v}\right)=\mathbb{P}_{1}\left(k_{v}\right)$ by reduction modulo $v$, and in particular $\mathrm{PGL}_{2}\left(k_{v}\right)$ acts simply transitively on triples of pairwise distinct points on $\operatorname{lk}\left(*_{v}\right)$. We identify the boundary at infinity $\partial_{\infty} \mathbb{T}_{v}$ of $\mathbb{T}_{v}$ with $\mathbb{P}_{1}\left(K_{v}\right)$ by the unique homeomorphism from $\partial_{\infty} \mathbb{T}_{v}$ to $\mathbb{P}_{1}\left(K_{v}\right)$ such that the (continuous) extension to $\partial_{\infty} \mathbb{T}_{v}$ of the isometric action of $\mathrm{PGL}_{2}\left(K_{v}\right)$ on $\mathbb{T}_{v}$ corresponds to the projective action of $\mathrm{PGL}_{2}\left(K_{v}\right)$ on $\mathbb{P}_{1}\left(K_{v}\right)$.

Let $\Gamma_{v}=\mathrm{PGL}_{2}\left(R_{v}\right)$. The group $\Gamma_{v}$ is a lattice in the locally compact group $\mathrm{PGL}_{2}\left(K_{v}\right)$, called the modular group at $v$ of $K$. The quotient graph $\Gamma \backslash \mathbb{T}_{v}$ is called the modular graph of $K$, and the quotient graph of groups $\Gamma \rrbracket \mathbb{T}_{v}$ is called the modular graph of groups at $v$ of $K$. We refer to [Ser] for background information on these objects, and for instance to [Pau] for a geometric treatment when $K=\mathbb{F}_{q}(Y)$ and $v=v_{\infty}$.

Recall that the open horoballs centred at $\xi \in \partial_{\infty} \mathbb{T}_{v}$ are the subsets of the geometric realisation $\left|\mathbb{T}_{v}\right|$ of $\mathbb{T}_{v}$ defined by

$$
\mathscr{H}\left(\rho_{\xi}\right)=\left\{y \in\left|\mathbb{T}_{v}\right|: \lim _{t \rightarrow+\infty}\left(t-d\left(\rho_{\xi}(t), y\right)\right)>0\right\}
$$


where $\rho_{\xi}$ is a geodesic ray converging to $\xi$. The boundary of $\mathscr{H}\left(\rho_{\xi}\right)$ is the horosphere

$$
\partial \mathscr{H}\left(\rho_{\xi}\right)=\left\{y \in\left|\mathbb{T}_{v}\right|: \lim _{t \rightarrow+\infty}\left(t-d\left(\rho_{\xi}(t), y\right)\right)=0\right\} .
$$

We refer to $[\mathrm{BH}]$ for background on these notions. The height in $\mathscr{H}\left(\rho_{\xi}\right)$ of a point $x \in\left|\mathbb{T}_{v}\right|$ is $\lim _{t \rightarrow+\infty}\left(t-d\left(\rho_{\xi}(t), x\right)\right)$. It is positive if and only if $x$ belongs to $\mathscr{H}\left(\rho_{\xi}\right)$. We denote by $\mathscr{H}_{\infty}$ the unique horoball centred at $\infty \in \partial_{\infty} \mathbb{T}_{v}$ whose associated horosphere passes through $*_{v}$.

Let $\Gamma$ be a finite index subgroup of $\Gamma_{v}$. By for instance [Ser, Pau], there exists a $\Gamma$ equivariant family of pairwise disjoint open horoballs $\left(\mathscr{H}_{\xi}\right)_{\xi \in \mathbb{P}_{1}(K)}$ in $\mathbb{T}_{v}$ with $\mathscr{H}_{\xi}$ centered at $\xi$ and the stabiliser $\Gamma_{\xi}$ of $\xi$ in $\Gamma$ acting transitively on the boundary of $\mathscr{H}_{\xi}$ for every $\xi \in \mathbb{P}_{1}(K) \subset \partial_{\infty} \mathbb{T}_{v}$, so that the quotient by $\Gamma$ of

$$
\widetilde{E}_{\Gamma}=\mathbb{T}_{v}-\bigcup_{\xi \in \mathbb{P}_{1}(K)} \mathscr{H}_{\xi}
$$

is a finite connected graph, denoted by $E_{\Gamma}$. The set of cusps $\Gamma \backslash \mathbb{P}_{1}(K)$ is finite. For every representative $\xi$ of a cusp in $\Gamma \backslash \mathbb{P}_{1}(K)$, the injective image by the canonical projection $\mathbb{T}_{v} \rightarrow \Gamma \backslash \mathbb{T}_{v}$ of any geodesic ray starting from a point on the boundary of $\mathscr{H}_{\xi}$ with point at infinity $\xi$ is called a cuspidal ray. The quotient graph $\Gamma \backslash \mathbb{T}_{\infty}$ is the union of the finite subgraph $E_{\Gamma}$ and the finite collection of cuspidal rays that are glued to $E_{\Gamma}$ at their origin.

Example 2.2. (See for instance $[\mathrm{BaL}]$.) Let $K=\mathbb{F}_{q}(Y)$ and $v=v_{\infty}$. Then $\Gamma_{v_{\infty}}=$ $\mathrm{PGL}_{2}\left(\mathbb{F}_{q}[Y]\right)$ and the quotient graph of groups $\Gamma_{v_{\infty}} \| \mathbb{T}_{v_{\infty}}$ is the following modular ray

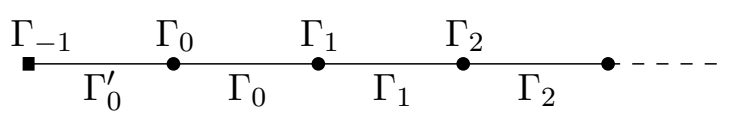

where $\Gamma_{-1}=\operatorname{PGL}_{2}\left(\mathbb{F}_{q}\right), \Gamma_{0}^{\prime}=\Gamma_{0} \cap \Gamma_{-1}$ and, for every $n \in \mathbb{N}$,

$$
\Gamma_{n}=\left\{\left[\begin{array}{ll}
a & b \\
0 & d
\end{array}\right] \in \mathrm{PGL}_{2}\left(\mathbb{F}_{q}[Y]\right): a, d \in \mathbb{F}_{q}^{\times}, b \in \mathbb{F}_{q}[Y], \operatorname{deg} b \leq n+1\right\} .
$$

The canonical $\Gamma_{v_{\infty}}$-equivariant family $\left(\mathscr{H}_{\xi}\right)_{\xi \in \mathbb{P}_{1}(K)}$ of pairwise disjoint maximal open horoballs in $\mathbb{T}_{v_{\infty}}$, with $\mathscr{H}_{\xi}$ centered at $\xi$ for every $\xi \in \mathbb{P}_{1}(K)$, consists of the connected components of $\left|\mathbb{T}_{v_{\infty}}\right|-\Gamma_{v_{\infty}} *_{v_{\infty}}$. The graph $\widetilde{E}_{\Gamma}$ is reduced to the orbit of the base point $*_{v_{\infty}}$, and $E_{\Gamma}$ is reduced to one vertex, the origin of the modular ray (see the above picture). In particular, the diameter of $E_{\Gamma}$ is zero.

All geodesic lines in $\mathbb{T}_{v_{\infty}}$ pass through the $\Gamma_{v_{\infty}}$-orbit of $*_{v_{\infty}}$. Indeed, no geodesic is completely contained in a horoball and since $\bigcup_{\xi \in \mathbb{P}_{1}(K)} \partial \mathscr{H}_{\xi}=\Gamma_{v_{\infty}} *_{v_{\infty}}$, the only way a geodesic line exits a horoball of the canonical family $\left(\mathscr{H}_{\xi}\right)_{\xi \in \mathbb{P}_{1}(K)}$ is through the orbit of $*_{v_{\infty}}$. The intersection of a geodesic line with the orbit $\Gamma_{v_{\infty}} *_{v_{\infty}}$ is hence finite if and only if its endpoints are both in $\mathbb{P}_{1}(K)$.

We end this section with the following lemma, which is an effective version of a special case of [HP, Prop. 2.6]. It controls the intersection length of a translation axis of an element of a discrete group of automorphisms of a tree with its images under this group. We will use it in Section 3 in order to prove Theorem 3.4. 
Recall that an automorphism $\gamma$ of a simplicial tree $\mathbb{T}$ with geometric realisation $|\mathbb{T}|$ is loxodromic if it fixes no point of $|\mathbb{T}|$, that its translation length $\ell(\gamma)=\min _{x \in V \mathbb{T}} d(x, \gamma x)$ is then positive and that its translation axis $\operatorname{Ax}_{\gamma}=\{x \in|\mathbb{T}|: d(x, \gamma x)=\ell(\gamma)\}$ is then a geodesic line in $|\mathbb{T}|$.

Lemma 2.3. Let $\Gamma$ be a discrete group of automorphisms of a locally finite tree $\mathbb{T}$. Let $\gamma_{0} \in \Gamma$ be a loxodromic element on $\mathbb{T}$. Let $k_{0}=\min _{x \in \mathrm{Ax}_{\gamma_{0}}}\left|\Gamma_{x}\right|$ be the minimal order of the stabiliser in $\Gamma$ of a vertex of $\mathrm{Ax}_{\gamma_{0}}$ and let $\Gamma_{0}$ be the stabiliser of $\mathrm{Ax}_{\gamma_{0}}$ in $\Gamma$. Then for every $\gamma \in \Gamma-\Gamma_{0}$, the length of the geodesic segment $\gamma \mathrm{Ax}_{\gamma_{0}} \cap \mathrm{Ax}_{\gamma_{0}}$ is less than $\left(k_{0}+1\right) \ell\left(\gamma_{0}\right)-1$.

Proof. Assume for a contradiction that the length $L \in \mathbb{N}$ of $\gamma \mathrm{Ax}_{\gamma_{0}} \cap \mathrm{Ax}_{\gamma_{0}}$ is at least $\left(k_{0}+1\right) \ell\left(\gamma_{0}\right)-1$. Denote by $[x, y]$ the geodesic segment $\gamma \mathrm{Ax}_{\gamma_{0}} \cap \mathrm{Ax}_{\gamma_{0}}$, such that $\gamma_{0} x$ and $y$ are on same side of $x$ on $A_{x_{0}}$. Let $\epsilon=1$ if $\gamma \gamma_{0} \gamma^{-1} x$ and $y$ are on same side of $x$ on $\gamma \mathrm{Ax}_{\gamma_{0}}$, and $\epsilon=-1$ otherwise.

Since $\gamma_{0}$ acts by a translation of length $\ell\left(\gamma_{0}\right)$ on $\mathrm{Ax}_{\gamma_{0}}$, there exists a point $x^{\prime} \in[x, y]$ at distance at most $\ell\left(\gamma_{0}\right)-1$ from $x$ such that $\left|\Gamma_{x^{\prime}}\right|=k_{0}$. Note that $\gamma \gamma_{0}^{\epsilon} \gamma^{-1}$ acts by a translation of length $\ell\left(\gamma_{0}\right)$ on $\gamma \mathrm{Ax}_{\gamma_{0}}$ and that the translation directions of $\gamma_{0}$ and $\gamma \gamma_{0}^{\epsilon} \gamma^{-1}$ coincide on $[x, y]$. Hence for every $k \in\left\{0,1, \ldots, k_{0}\right\}$, the point $\gamma \gamma_{0}^{\epsilon k} \gamma^{-1} x^{\prime}$ belongs to $[x, y]$ by the assumption on $L$, and $\gamma_{0}^{-k} \gamma \gamma_{0}^{\epsilon k} \gamma^{-1} x^{\prime}=x^{\prime}$. Since the stabiliser of $x^{\prime}$ has order less than $k_{0}+1$, there are hence distinct $k, k^{\prime} \in\left\{0,1, \ldots, k_{0}\right\}$ such that

$$
\gamma_{0}^{-k} \gamma \gamma_{0}^{\epsilon k} \gamma^{-1}=\gamma_{0}^{-k^{\prime}} \gamma \gamma_{0}^{\epsilon k^{\prime}} \gamma^{-1}
$$

that is, $\gamma_{0}^{k^{\prime}-k} \gamma=\gamma \gamma_{0}^{\epsilon\left(k^{\prime}-k\right)}$. Since $\mathrm{Ax}_{\gamma_{0}^{m}}=\mathrm{Ax}_{\gamma_{0}}$ and $\gamma \mathrm{Ax}_{\gamma_{0}{ }^{\prime}}=\mathrm{Ax}_{\gamma \gamma_{0}{ }^{m^{\prime}} \gamma^{-1}}$ for all $m, m^{\prime} \in$ $\mathbb{Z}-\{0\}$, this implies that $\gamma$ preserves $\mathrm{Ax}_{\gamma_{0}}$, a contradiction since $\gamma \notin \Gamma_{0}$.

\section{Quadratic Diophantine approximation in completions of function fields}

Let $K$ be a function field over $\mathbb{F}_{q}$, let $v$ be a (normalised discrete) valuation of $K$, let $R_{v}$ be the affine function ring associated with $v$, and let $\Gamma$ be a finite index subgroup of $\Gamma_{v}=\mathrm{PGL}_{2}\left(R_{v}\right)$ (for instance a congruence subgroup).

We denote by

$$
\mathrm{QI}_{v}=\left\{x \in K_{v}:[K(x): K]=2\right\}
$$

the set of quadratic irrationals in $K_{v}$ over $K$, and we fix $\alpha \in \mathrm{QI}_{v}$. We denote by $\alpha^{\sigma} \in \mathrm{QI}_{v}$ the Galois conjugate of $\alpha$ over $K$, and by

$$
\Theta_{\alpha, \Gamma}=\Gamma \cdot\left\{\alpha, \alpha^{\sigma}\right\}
$$

the union of the orbits of $\alpha$ and $\alpha^{\sigma}$ under the projective action of $\Gamma$, with $\Theta_{\alpha}=\Theta_{\alpha, \Gamma_{v}}$. Note that $\alpha^{\sigma} \neq \alpha$, since an irreducible quadratic polynomial over $K$ which is inseparable does not split over $K_{v}$, and that there exists a loxodromic element $\gamma_{\alpha} \in \Gamma_{v}$ such that ]$\alpha, \alpha^{\sigma}\left[=\operatorname{Ax}_{\gamma_{\alpha}}\right.$ (see for instance [BPP, Lem. 17.2]). For all $x \in K_{v}$ and $\beta \in \mathrm{QI}_{v}$ with $x \neq \beta$, let

$$
c(x, \beta)=\frac{|x-\beta|_{v}}{\left|\beta-\beta^{\sigma}\right|_{v}} \in q_{v}^{\mathbb{Z}} .
$$

The following result gives a geometric interpretation to this quantity. 
Lemma 3.1. Let $x \in K_{v}$ and $\beta \in \mathrm{QI}_{v}$ with $x \neq \beta, \beta^{\sigma}$.

(1) If the geodesic lines $] \infty, x[$ and $] \beta, \beta^{\sigma}\left[\right.$ in $\mathbb{T}_{v}$ are disjoint or meet at only one vertex, then, with $n$ the distance between them,

$$
c(x, \beta)=c\left(x, \beta^{\sigma}\right)=q_{v}^{n} \geq 1 .
$$

(2) If the geodesic lines $] \infty, x[$ and $] \beta, \beta^{\sigma}\left[\right.$ in $\mathbb{T}_{v}$ meet along a geodesic segment of length $n>0$, with the closest point to $\beta$ on $] \infty, x\left[\right.$ closer to $x$ than the closest point to $\beta^{\sigma}$ on ]$\infty, x[$, then

$$
c(x, \beta)=q_{v}^{-n}<1=c\left(x, \beta^{\sigma}\right) .
$$

In particular, $\min \left\{c(x, \beta), c\left(x, \beta^{\sigma}\right)\right\}<1$.
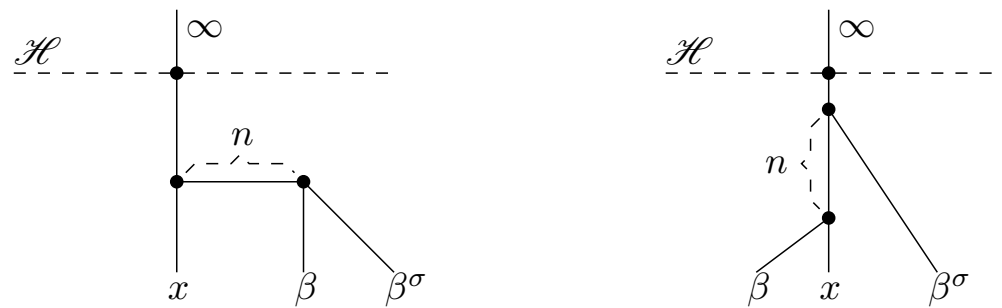

Proof. For all distinct $y, z \in K_{v}$ and for every horoball $\mathscr{H}$ in $\mathbb{T}_{v}$ centered at $\infty$, let

$$
d_{\mathscr{H}}(y, z)=\lim _{t \rightarrow+\infty} e^{\frac{1}{2} d\left(y_{t}, z_{t}\right)-t},
$$

where $t \mapsto y_{t}, t \mapsto z_{t}$ are the geodesic lines starting from $\infty$, through $\partial \mathscr{H}$ at time $t=0$, ending at the points at infinity $y, z$ respectively. By for instance [BPP, Eq. (15.2)], we have

$$
|y-z|_{v}=d_{\mathscr{H}_{\infty}}(y, z)^{\ln q_{v}} .
$$

Since the ratio $\frac{d_{\mathscr{H}}(y, z)}{d_{\mathscr{H}}\left(y^{\prime}, z^{\prime}\right)}$ does not depend on the horoball $\mathscr{H}$ centered at $\infty$, if $\mathscr{H}$ is small enough, we hence have

$$
c(x, \beta)=\frac{|x-\beta|_{v}}{\left|\beta-\beta^{\sigma}\right|_{v}}=q_{v}^{-d(\mathscr{H},] x, \beta[)+d(\mathscr{H},] \beta, \beta^{\sigma}[) .}
$$

The result follows.

For every $x \in K_{v}-\left(K \cup \Theta_{\alpha, \Gamma}\right)$, we define the approximation constant of $x$ by the (extended) $\Gamma$-orbit of $\alpha$ as

$$
c_{\alpha, \Gamma}(x)=\liminf _{\beta \in \Theta_{\alpha, \Gamma},\left|\beta-\beta^{\sigma}\right|_{v} \rightarrow 0} c(x, \beta) .
$$

When $x$ is itself a quadratic irrational, the following result gives a geometric computation of the approximation constant $c_{\alpha, \Gamma}(x)$.

Remark 3.2. For all $\alpha, \beta \in \mathrm{QI}_{v}$ such that $\beta \notin \Theta_{\alpha, \Gamma}$, we have

$$
c_{\beta, \Gamma}(\alpha)=c_{\alpha, \Gamma}(\beta)=q^{-n(\alpha, \beta)}
$$

where $n(\alpha, \beta)=\max _{\gamma \in \Gamma}$ length (]$\alpha^{\sigma}, \alpha[\cap \gamma] \beta^{\sigma}, \beta[)$ if some image of $] \beta^{\sigma}, \beta[$ by an element of $\Gamma$ meets $] \alpha^{\sigma}, \alpha\left[\right.$, and $n(\alpha, \beta)=-\min _{\gamma \in \Gamma} d(] \alpha^{\sigma}, \alpha[, \gamma] \beta^{\sigma}, \beta[)$ otherwise. 
Proof. Note that since the elements of $\Gamma$ preserve the lengths of segments, and by a change of variable $\gamma \mapsto \gamma^{-1}$, we have $n(\beta, \alpha)=n(\alpha, \beta)$, so that the first equality follows from the second one.

By a proof similar to the one of Proposition 2.3, for all $\alpha, \beta \in \mathrm{QI}_{v}$ such that $\beta \notin \Theta_{\alpha, \Gamma}$, there exists a constant $\ell_{\alpha, \beta}$ (depending only on the translation lengths of primitive elements in $\Gamma$ preserving the geodesic lines $] \alpha^{\sigma}, \alpha[$ and $] \beta^{\sigma}, \beta[$, as well as on the cardinalities of the stabilisers in $\Gamma$ of the vertices of these geodesic lines) such that for every $\gamma \in \Gamma$, the length of the intersection $] \alpha^{\sigma}, \alpha[\cap \gamma] \beta^{\sigma}, \beta\left[\right.$ is at most $\ell_{\alpha, \beta}$.

First assume that some image of $] \beta^{\sigma}, \beta[$ by an element of $\Gamma$ meets $] \alpha^{\sigma}, \alpha[$. Using Lemma 3.1 for the second equality, the fact that $] \infty, \beta[$ and $] \beta^{\sigma}, \beta[$ share a subray with endpoint $\beta$ for the third equality, and Lemma 3.1 (2) for the fourth one, we have

$$
\begin{aligned}
& c_{\alpha, \Gamma}(\beta)=\liminf _{\alpha^{\prime} \in \Theta_{\alpha, \Gamma},\left|\alpha^{\prime \sigma}-\alpha^{\prime}\right|_{v} \rightarrow 0} c\left(\beta, \alpha^{\prime}\right)=\liminf _{\left.\alpha^{\prime} \in \Theta_{\alpha, \Gamma},\left|\alpha^{\prime}-\alpha^{\prime}\right|_{v} \rightarrow 0,\right] \alpha^{\prime}, \alpha^{\prime}[\cap] \infty, \beta[\neq \emptyset} c\left(\beta, \alpha^{\prime}\right) \\
& =\liminf _{\left.\alpha^{\prime} \in \Theta_{\alpha, \Gamma},\left|\alpha^{\prime \sigma}-\alpha^{\prime}\right|_{v} \rightarrow 0,\right] \alpha^{\prime \sigma}, \alpha^{\prime}[\cap] \beta^{\sigma}, \beta[\neq \emptyset} c\left(\beta, \alpha^{\prime}\right) \\
& =\liminf _{\left.\alpha^{\prime} \in \Theta_{\alpha, \Gamma},\left|\alpha^{\prime \sigma}-\alpha^{\prime}\right|_{v} \rightarrow 0,\right] \alpha^{\prime \sigma}, \alpha^{\prime}[\cap] \beta^{\sigma}, \beta[\neq \emptyset} q^{-\operatorname{length}(] \alpha^{\prime \sigma}, \alpha^{\prime}[\cap] \beta^{\sigma}, \beta[)}=q^{-n(\beta, \alpha)} .
\end{aligned}
$$

Otherwise, the result follows by using Lemma 3.1 (1).

We define the quadratic Lagrange spectrum of $\alpha$ relative to $\Gamma$ as

$$
\operatorname{Sp}(\alpha, \Gamma)=\left\{c_{\alpha, \Gamma}(x): x \in K_{v}-\left(K \cup \Theta_{\alpha, \Gamma}\right)\right\},
$$

and $\operatorname{Sp}(\alpha)=\operatorname{Sp}\left(\alpha, \Gamma_{v}\right)$. Note that $\operatorname{Sp}(\alpha, \Gamma)$ is contained in $q_{v}^{\mathbb{Z}} \cup\{0,+\infty\}$ and that for every $\beta \in \Theta_{\alpha, \Gamma}$, the functions $c_{\alpha, \Gamma}$ and $c_{\beta, \Gamma}$ on $K_{v}-\left(K \cup \Theta_{\alpha, \Gamma}\right)=K_{v}-\left(K \cup \Theta_{\beta, \Gamma}\right)$ coincide, so that $\operatorname{Sp}(\alpha, \Gamma)=\operatorname{Sp}(\beta, \Gamma)$.

Proposition 3.3. The quadratic Lagrange spectrum $\operatorname{Sp}(\alpha, \Gamma)$ is closed and bounded in $\mathbb{R}$.

The above result allows us to define the Hurwitz constant of $\alpha$ relative to $\Gamma$ as

$$
\max \operatorname{Sp}(\alpha, \Gamma) \in q_{v}^{\mathbb{Z}},
$$

and the Hurwitz constant of $\alpha$ as $\max \operatorname{Sp}\left(\Gamma_{v}, \alpha\right)$. The proof of Proposition 3.3 actually gives an upper bound on $\operatorname{Sp}(\alpha, \Gamma)$ which does not depend on the quadratic irrational $\alpha$, see Equation (1) below. In the special case when $(K, v, \Gamma)=\left(\mathbb{F}_{q}(Y), v_{\infty}, \Gamma_{v_{\infty}}\right)$, we will prove more precisely in Section 4 that $\max _{\alpha \in \mathrm{QI}_{v}} \max \operatorname{Sp}(\alpha)=\frac{1}{q^{2}}$.

Proof. It follows from [HP, Thm. 1.6 $]^{4}$ that if $m_{K_{v}}$ is a Haar measure on the locally compact additive group of $K_{v}$, then $c_{\alpha, \Gamma}(x)=0$ for $m_{K_{v}}$-almost every $x \in K_{v}$. Therefore $0 \in \operatorname{Sp}(\alpha, \Gamma)$, and the quadratic Lagrange spectrum of $\alpha$ relative to $\Gamma$ is closed.

Let us fix $x \in K_{v}-K$ and let us prove that $c_{\alpha, \Gamma}(x) \leq q_{v}^{\operatorname{diam}} E_{\Gamma}$, where $E_{\Gamma}$ is as defined in Section 2. This proves Proposition 3.3 with a uniform bound on the Hurwitz constants

$$
\forall \alpha \in \mathrm{QI}_{v}, \quad \max \operatorname{Sp}(\alpha, \Gamma) \leq q_{v}^{\operatorname{diam}} E_{\Gamma} .
$$

\footnotetext{
${ }^{4}$ Actually, Thm. 1.6 of [HP] is stated only for $K=\mathbb{F}_{q}(Y), v=v_{\infty}$ and $\Gamma=\Gamma_{v}$, but it has an analogous version for general $(K, v, \Gamma)$ by using [HP, Prop. 1.5].
} 
Since $x$ is irrational and since any geodesic ray entering into a horoball and not converging towards its point at infinity has to exit the horoball, the geodesic line $] \infty, x[$ from $\infty$ to $x$ cannot stay after a given time in a given horoball of the family $\left(\mathscr{H}_{\xi}\right)_{\xi \in \mathbb{P}_{1}(K)}$ defined in Section 2. Hence there exists a sequence $\left(p_{n}\right)_{n \in \mathbb{N}}$ of points of $\widetilde{E}_{\Gamma}$ converging to $x$ along the geodesic line $] \infty, x\left[\right.$. Since $E_{\Gamma}=\Gamma \backslash \widetilde{E}_{\Gamma}$ is finite and since no geodesic line is contained in a horoball, there exists a sequence $\left(\gamma_{n}^{\prime}\right)_{n \in \mathbb{N}}$ in $\Gamma$ such that $d\left(p_{n}, \gamma_{n}^{\prime}\right] \alpha, \alpha^{\sigma}[) \leq \operatorname{diam} E_{\Gamma}$ for all $n \in \mathbb{N}$.

By Lemma 3.1, there exists $\beta_{n} \in\left\{\gamma_{n}^{\prime} \alpha, \gamma_{n}^{\prime} \alpha^{\sigma}\right\} \subset \Theta_{\alpha, \Gamma}$ such that $c\left(x, \beta_{n}\right)<1$ if ]$\beta_{n}, \beta_{n}^{\sigma}\left[=\gamma_{n}^{\prime}\right] \alpha, \alpha^{\sigma}[$ meets $] \infty, x\left[\right.$ in at least an edge, and $c\left(x, \beta_{n}\right) \leq q_{v}^{\text {diam } E_{\Gamma}}$ otherwise. Hence

$$
\liminf _{n \rightarrow+\infty} c\left(x, \beta_{n}\right) \leq q_{v}^{\operatorname{diam} E_{\Gamma}} .
$$

Let $\gamma_{\alpha} \in \Gamma_{v}$ be a loxodromic element such that $] \alpha, \alpha^{\sigma}\left[=\operatorname{Ax}_{\gamma_{\alpha}}\right.$. Since $\Gamma$ has finite index in $\Gamma_{v}$, up to replacing $\gamma_{\alpha}$ by a positive power, we may assume that $\gamma_{\alpha}$ belongs to $\Gamma$. Since the length of the intersection of two distinct translates of $\mathrm{Ax}_{\gamma_{\alpha}}$ by elements of $\Gamma$ is uniformly bounded by Lemma 2.3, we have $\lim _{n \rightarrow+\infty}\left|\beta_{n}-\beta_{n}^{\sigma}\right|_{v}=0$. Hence by the definition of the approximation constants, we have as wanted $c_{\alpha, \Gamma}(x) \leq q_{v}^{\text {diam } E_{\Gamma}}$.

The following result, which implies Theorem 1.1 (2) in the introduction, says that the nonarchimedean quadratic Lagrange spectra contain Hall rays. Note that its proof gives an explicit upper bound on the constant whose existence is claimed.

Theorem 3.4. There exists $m_{\alpha} \in \mathbb{N}$ such that for all $m \in \mathbb{N}$ with $m \geq m_{\alpha}$, we have $q_{v}^{-m} \in \operatorname{Sp}(\alpha, \Gamma)$.

Proof. Let

$$
k_{\alpha}=\min _{x \in] \alpha^{\sigma}, \alpha\left[\cap V \mathbb{T}_{v}\right.}\left|\Gamma_{x}\right|
$$

be the minimal order of the stabiliser in $\Gamma$ of a vertex of the geodesic line $] \alpha^{\sigma}, \alpha\left[\right.$. Let $\gamma_{\alpha} \in \Gamma$ be a loxodromic element (with minimal translation length) such that $\left.\mathrm{Ax}_{\gamma_{\alpha}}=\right] \alpha^{\sigma}, \alpha[$ and let $\kappa_{\alpha}=\left(k_{\alpha}+1\right) \ell\left(\gamma_{\alpha}\right)-2$. By Lemma 2.3, for all $\beta, \beta^{\prime} \in \Theta_{\alpha, \Gamma}$, if $\beta^{\prime} \notin\left\{\beta, \beta^{\sigma}\right\}$, then the intersection $] \beta^{\sigma}, \beta[\cap] \beta^{\prime \sigma}, \beta^{\prime}$ [ is a (possibly empty) segment of length at most $\kappa_{\alpha}$. Take

$$
m_{\alpha}=2 \kappa_{\alpha}+1
$$

Let us fix $m \in \mathbb{N}$ with $m \geq m_{\alpha}$, and let us prove that $q_{v}^{-m}$ belongs to $\operatorname{Sp}(\alpha, \Gamma)$, which gives Theorem 3.4. For this, let us construct $\xi \in K_{v}-\left(K \cup \Theta_{\alpha, \Gamma}\right)$ and a sequence $\left(\beta_{n}\right)_{n \in \mathbb{N}}$ in $\Theta_{\alpha, \Gamma}$ such that

- $\left|\beta_{n}-\beta_{n}^{\sigma}\right|_{v} \rightarrow 0$ as $n \rightarrow+\infty$,

- the length of the intersection $] \beta_{n}^{\sigma}, \beta_{n}[\cap] \infty, \xi[$ is exactly $m$,

- the closest point to $\beta_{n}$ on ] $\infty, \xi$ [ is closer to $\xi$ than the closest point to $\beta_{n}^{\sigma}$ on ] $\infty, \xi[$,

- for every $\beta \in \Theta_{\alpha, \Gamma}$, either $] \beta^{\sigma}, \beta[$ and $] \infty, \xi[$ are disjoint or their nonempty intersection has length at most $m$.

By Lemma 3.1, this proves that

$$
c_{\alpha, \Gamma}(\xi)=\liminf _{\beta \in \Theta_{\alpha, \Gamma},\left|\beta-\beta^{\sigma}\right|_{v} \rightarrow 0} c(\xi, \beta)=\liminf _{n \rightarrow+\infty} c\left(\xi, \beta_{n}\right)=q_{v}^{-m},
$$

so that $q_{v}^{-m}$ does belong to $\operatorname{Sp}(\alpha, \Gamma)$. 
Since the image of $] \alpha^{\sigma}, \alpha\left[\right.$ in $\Gamma \backslash \mathbb{T}_{v}$ is compact, and since $\Gamma \cdot \infty$ is a cusp, there exists $\beta_{0} \in \Theta_{\alpha, \Gamma}$ such that if $x_{0}$ is the closest point to $\infty$ on $] \beta_{0}^{\sigma}, \beta_{0}[$, then the open geodesic ray ]$x_{0}, \infty[$ does not meet any $] \beta^{\sigma}, \beta\left[\right.$ for $\beta \in \Theta_{\alpha, \Gamma}$. Let $y_{0}$ be the vertex on $\left[x_{0}, \beta_{0}\right.$ [ at distance exactly $m$ from $x_{0}$, and let $e_{0}$ be an edge with origin $y_{0}$ and not contained in $] \beta_{0}^{\sigma}, \beta_{0}[$.
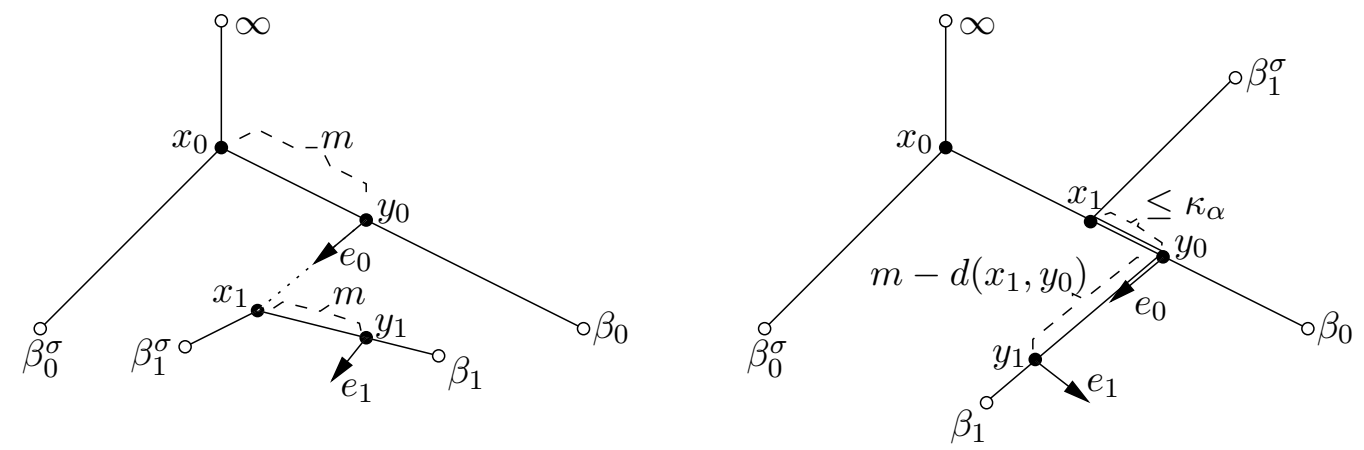

Assume first (see the above picture on the left) that there exists no $\beta \in \Theta_{\alpha, \Gamma}$ such that $e_{0}$ is contained in $] \beta^{\sigma}, \beta\left[\right.$. Since $\Gamma$ is a lattice, the set $\Theta_{\alpha, \Gamma}$, which contains the orbit $\Gamma \cdot \alpha$, is dense in $\partial_{\infty} \mathbb{T}_{v}$. Hence there exists $\beta_{1} \in \Theta_{\alpha, \Gamma}$ such that if $x_{1}$ is the closest point to $y_{0}$ on $] \beta_{1}^{\sigma}, \beta_{1}\left[\right.$, then the open segment ] $y_{0}, x_{1}$ [ contains $e_{0}$ in its closure and meets no ]$\beta^{\sigma}, \beta$ [ for $\beta \in \Theta_{\alpha, \Gamma}$. Let $y_{1}$ be the vertex on the geodesic ray $\left[x_{1}, \beta_{1}\right.$ [ at distance equal to $m$ from $x_{1}$, and let $e_{1}$ be an edge with origin $y_{1}$ and not contained in $] \beta_{1}^{\sigma}, \beta_{1}[$. Note that $d\left(y_{0}, y_{1}\right) \geq d\left(x_{1}, y_{1}\right)=m \geq m_{\alpha} \geq \kappa_{\alpha}+1$. By Lemma 2.3, for every $\beta \in \Theta_{\alpha, \Gamma}$, the length of the (possibly empty) intersection $\left[y_{1}, \infty[\cap] \beta^{\sigma}, \beta[\right.$ is at most $m$ (exactly $m$ if $\beta \in\left\{\beta_{0}, \beta_{0}^{\sigma}, \beta_{1}, \beta_{1}^{\sigma}\right\}$ and at most $\kappa_{\alpha}$ otherwise).

Assume now (see the above picture on the right) that there exists $\beta_{1} \in \Theta_{\alpha, \Gamma}$ such that $e_{0}$ is contained in $] \beta_{1}^{\sigma}, \beta_{1}\left[\right.$. Up to exchanging $\beta_{1}$ and $\beta_{1}^{\sigma}$, we may assume that $e_{0}$ is contained in $\left[y_{0}, \beta_{1}[\right.$.

Let $x_{1} \in V \mathbb{T}_{v}$ be such that $\left[y_{0}, \infty\left[\cap\left[y_{0}, \beta_{1}^{\sigma}\left[=\left[y_{0}, x_{1}\right]\right.\right.\right.\right.$. Note that by Lemma 2.3, we have $d\left(x_{1}, y_{0}\right) \leq \kappa_{\alpha}$ and $x_{1} \in\left[y_{0}, x_{0}\left[\right.\right.$ since $d\left(x_{0}, y_{0}\right)=m \geq m_{\alpha}>\kappa_{\alpha}$. Let $y_{1}$ be the point at distance equal to $m-d\left(x_{1}, y_{0}\right)$ from $y_{0}$ on $\left[y_{0}, \beta_{1}\left[\right.\right.$. Note that $d\left(y_{0}, y_{1}\right) \geq \kappa_{\alpha}+1$ since $m \geq m_{\alpha}=2 \kappa_{\alpha}+1$, and in particular by Lemma 2.3 , there is no $\beta \in \Theta_{\alpha, \Gamma}$ such that $] \beta^{\sigma}, \beta[$ contains both $e_{1}$ and $y_{0}$. By Lemma 2.3, for every $\beta \in \Theta_{\alpha, \Gamma}$, the length of $\left[y_{1}, \infty[\cap] \beta^{\sigma}, \beta[\right.$ is at most $m$ (exactly $m$ if $\beta \in\left\{\beta_{0}, \beta_{0}^{\sigma}, \beta_{1}, \beta_{1}^{\sigma}\right\}$ and at most $2 \kappa_{\alpha}$ otherwise).

By iterating this construction, we obtain sequences $\left(\beta_{n}\right)_{n \in \mathbb{N}}$ in $\Theta_{\alpha, \Gamma},\left(x_{n}\right)_{n \in \mathbb{N}},\left(y_{n}\right)_{n \in \mathbb{N}}$ in $V \mathbb{T}_{v}$ and $\left(e_{n}\right)_{n \in \mathbb{N}}$ in $E \mathbb{T}_{v}$ such that, for all $n \in \mathbb{N}$,

- $y_{n} \in\left[y_{n+1}, \infty\left[\right.\right.$ and $d\left(y_{n}, y_{n+1}\right) \geq \kappa_{\alpha}+1$,

- for every $\beta \in \Theta_{\alpha, \Gamma}$, the length of $\left[y_{n}, \infty[\cap] \beta^{\sigma}, \beta[\right.$ is at most $m$, and the length of $\left[y_{n}, \infty[\cap] \beta_{n}^{\sigma}, \beta_{n}\left[=\left[y_{n}, x_{n}\right]\right.\right.$ is exactly $m$,

- $y_{n} \in\left[x_{n}, \beta_{n}[\right.$,

- $e_{n}$ exits $] \beta_{n}^{\sigma}, \beta_{n}\left[\right.$ at $y_{n}$ and is contained in $\left[y_{n}, y_{n+1}\right]$,

- $x_{n+1}$ and $y_{n}$ belong to $\left[x_{n}, y_{n+1}\right]$, and either $y_{n}$ belongs to $\left[x_{n}, x_{n+1}[\right.$ in which case ]$y_{n}, x_{n+1}[$ meets no $] \beta^{\sigma}, \beta\left[\right.$ for $\beta \in \Theta_{\alpha, \Gamma}$, or $y_{n}$ belongs to $\left[x_{n+1}, y_{n+1}[\right.$.

By the first point, the sequence $\left(y_{n}\right)_{n \in \mathbb{N}}$ converges to $\xi \in \partial_{\infty} \mathbb{T}_{v}$, such that $y_{0}, y_{1}, y_{2}, \ldots$ are in this order on the geodesic line ] $\infty, \xi$ [ oriented from $\infty$ to $\xi$. The point at infinity $\xi$ does not belong to $K$ (since $y_{n}$ belongs to $] \beta_{n}^{\sigma}, \beta_{n}$ [ and the geodesic lines ] $\beta^{\sigma}, \beta$ [ for $\beta \in \Theta_{\alpha, \Gamma}$ 
do not enter a small enough horoball centered at any point of $K$ ) or to $\Theta_{\alpha, \Gamma}$ (otherwise ]$\infty, \xi[\cap] \xi^{\sigma}, \xi$ [ would be infinitely long, contradicting the second point for $n$ large enough).

The length of $] \infty, \xi[\cap] \beta_{n}^{\sigma}, \beta_{n}\left[\right.$, which is equal to $\left[y_{n}, x_{n}\right]$ since $e_{n}$ exits $] \beta_{n}^{\sigma}, \beta_{n}\left[\right.$ at $y_{n}$, is exactly $m$, and in particular is bounded in $n$. Since $\left(y_{n}\right)_{n \in \mathbb{N}}$ converges to the point at infinity $\xi$, we have $\left|\beta_{n}-\beta_{n}^{\sigma}\right|_{v} \rightarrow 0$ as $n \rightarrow+\infty$. By construction, there is no $\beta \in \Theta_{\alpha, \Gamma}$ such that the length of $] \infty, \xi[\cap] \beta^{\sigma}, \beta[$ is larger than $m$.

Therefore $\xi$ satisfies the properties required at the beginning of the proof, and Theorem 3.4 follows.

In the next section, we will give several computations, using the continued fraction expansions, in the special case when $K=\mathbb{F}_{q}(Y), v=v_{\infty}$ is the valuation at infinity, and $\Gamma=\Gamma_{v_{\infty}}$ is the full Nagao lattice $\operatorname{PGL}_{2}\left(\mathbb{F}_{q}[Y]\right)$.

\section{Computations of approximation constants, Hurwitz con- stants and quadratic Lagrange spectra for fields of formal Laurent series}

In this section, we use the notation $\mathbb{F}_{q}, R=\mathbb{F}_{q}[Y], K=\mathbb{F}_{q}(Y), \widehat{K}=\mathbb{F}_{q}\left(\left(Y^{-1}\right)\right),|\cdot|$ given in the introduction. Let

$$
\mathscr{O}=\{f \in \widehat{K}:|f| \leq 1\}=\mathbb{F}_{q}\left[\left[Y^{-1}\right]\right]
$$

be the ring of formal power series in one variable $Y^{-1}$ over $\mathbb{F}_{q}$. Its unique maximal ideal is $\mathfrak{m}=Y^{-1} \mathscr{O}$. We denote by $\mathbb{T}$ the Bruhat-Tits tree of $\left(\mathrm{PGL}_{2}, \widehat{K}\right)$, with standard base point $*=[\mathscr{O} \times \mathscr{O}]$, and $\Gamma=\mathrm{PGL}_{2}(R)$, see Section 2 .

Any element $f \in \widehat{K}$ may be uniquely written as a sum $f=[f]+\{f\}$ of its integral part $[f] \in R$ and its fractional part $\{f\} \in \mathfrak{m}$. The Artin map $\Psi: \mathfrak{m}-\{0\} \rightarrow \mathfrak{m}$ is defined by $f \mapsto\left\{\frac{1}{f}\right\}$. Any $f \in \widehat{K}-K$ has a unique continued fraction expansion

$$
f=\left[a_{0}, a_{1}, a_{2}, a_{3}, \ldots\right]=a_{0}+\frac{1}{a_{1}+\frac{1}{a_{2}+\frac{1}{a_{3}+\ddots}}}
$$

with $a_{0}=a_{0}(f)=[f] \in R$, and $a_{n}=a_{n}(f)=\left[\frac{1}{\Psi^{n-1}\left(f-a_{0}\right)}\right] \in R$ a nonconstant polynomial for $n \geq 1$. The polynomials $a_{n}(f)$ are called the coefficients of the continued fraction expansion of $f$. For every $n \in \mathbb{N}$, the rational element

$$
\frac{P_{n}}{Q_{n}}=\left[a_{0}, a_{1}, a_{2}, \ldots, a_{n-a}, a_{n}\right]=a_{0}+\frac{1}{a_{1}+\frac{1}{a_{2}+\frac{1}{\ddots+\frac{\ddots}{a_{n-1}+\frac{1}{a_{n}}}}}}
$$


is the $n$-th convergent of $f$. We refer to [Las, Sch, Pau] for details and further information on continued fraction expansions of formal Laurent series and their geometric interpretation in terms of the Bruhat-Tits tree $\mathbb{T}$.

For every $f \in \widehat{K}-K$, let

$$
\begin{aligned}
& M(f)=\limsup _{k \rightarrow+\infty} \operatorname{deg} a_{k} \geq 1, \\
& M_{2}(f)=\limsup _{k \rightarrow+\infty}\left(\operatorname{deg} a_{k}+\operatorname{deg} a_{k+1}\right) \geq 2, \\
& m(f)=\liminf _{k \rightarrow+\infty} \operatorname{deg} a_{k} \geq 1 .
\end{aligned}
$$

As recalled in the introduction, an irrational element $\alpha \in \widehat{K}-K$ is quadratic over $K$ if and only if its continued fraction expansion is eventually periodic: For every $p \in$ $\mathbb{N}$ large enough, the sequence of coefficients $\left(a_{k+p}(\alpha)\right)_{k \in \mathbb{N}}$ is periodic with period $m \in$ $\mathbb{N}-\{0\}$ and, as usual, we then write the continued fraction expansion of $\alpha$ as $\alpha=$ $\left[a_{0}, a_{1}, a_{2}, \ldots, a_{p-1}, \overline{a_{p}, a_{p+1}, \ldots, a_{p+m-1}}\right]$. We then have

$$
M(\alpha)=\max _{0 \leq k \leq m-1} \operatorname{deg} a_{p+k}(\alpha) \quad \text { and } m(\alpha)=\min _{0 \leq k \leq m-1} \operatorname{deg} a_{p+k}(\alpha) .
$$

Let us recall from [Pau, $\S 6.3$, Rem. 2] the penetration properties of the geodesic lines of $\mathbb{T}$ inside the canonical equivariant family of pairwise disjoint open horoballs whose closures cover $\mathbb{T}$, see the picture below. For every $f \in \widehat{K}-K$, the geodesic line $] \infty, f[$ (oriented from $\infty$ to $f$ ) starts in $H_{0}(f)=\mathscr{H}_{\infty}$ by an initial negative subray; after $\mathscr{H}_{\infty}$, it successively passes through an infinite sequence of open horoballs in the family $\left(\mathscr{H}_{\xi}\right)_{\xi \in \mathbb{P}^{1}(K)}$, denoted by $\left(H_{n}(f)\right)_{n \in \mathbb{N}-\{0\}}$ and for every $n \in \mathbb{N}$, we have $H_{n+1}(f)=\mathscr{H}_{P_{n} / Q_{n}}$, where $P_{n} / Q_{n}$ is the $n$-th convergent of $f$. For every $n \in \mathbb{N}$, the maximum height of a point of $] \infty, f$ [inside $H_{n+1}(f)$ is equal to $\operatorname{deg} a_{n+1}$, or, equivalently, the intersection of $H_{n+1}(f)$ with $] \infty, f[$ is an open segment of length $2 \operatorname{deg} a_{n+1}$.

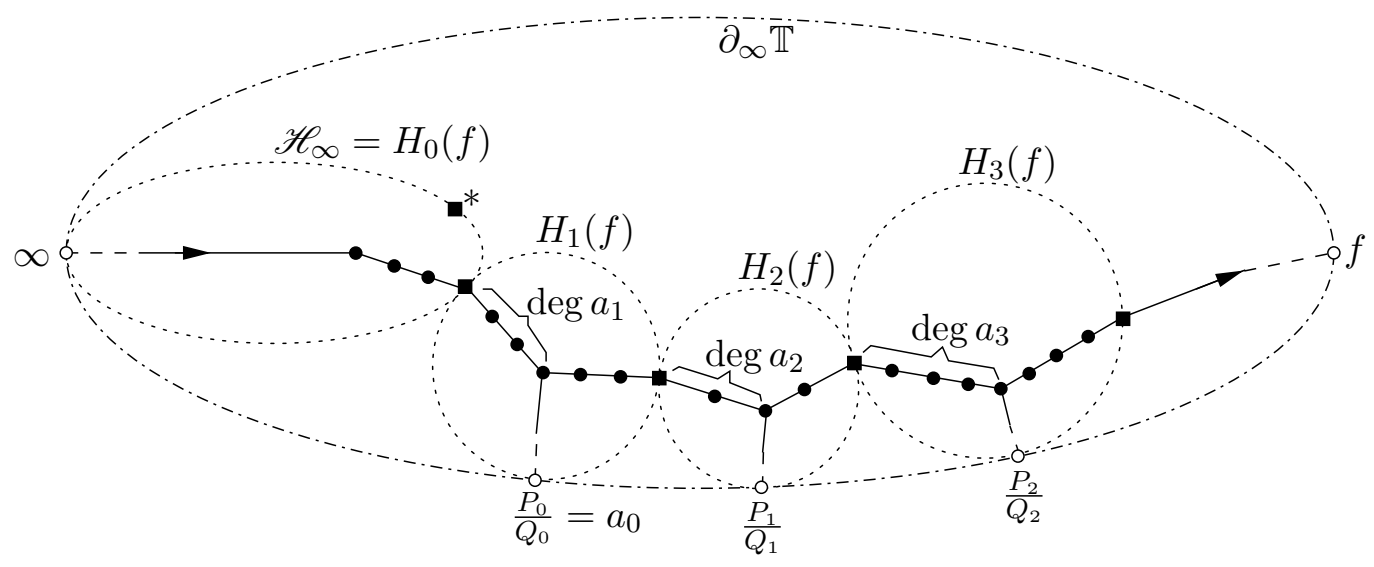

We will need the following lemmas, which also follow from the geometric interpretation of the continued fraction expansion given in [Pau], in order to estimate the quadratic approximation constants of elements $f \in \widehat{K}-K$.

Lemma 4.1. Let $f, f^{\prime} \in \widehat{K}-K$ with $a_{0}(f)=a_{0}\left(f^{\prime}\right)$. If the geodesic lines $] \infty, f[$ and ]$\infty, f^{\prime}\left[\right.$ coincide inside the horoball $\mathscr{H}_{a_{0}(f)}$, then the polynomial $a_{1}(f)-a_{1}\left(f^{\prime}\right)$ is constant. 
Lemma 4.2. Let $f \in \widehat{K}-K$ and $\alpha \in \mathrm{QI}$ with purely periodic continued fraction expansion. If there exist $p, q \in \mathbb{N}$ and $m \in \mathbb{N}$ such that $a_{p+1}(f)=a_{q+1}(\alpha), \ldots, a_{p+m}(f)=a_{q+m}(\alpha)$, then there exists $\gamma \in \Gamma$ such that the geodesic lines $] \infty, f[$ and $\gamma] \alpha^{\sigma}, \alpha[$ coincide at least in $H_{p+1}(f) \cup \cdots \cup H_{p+m}(f)$, so that their intersection has length at least

$$
2 \sum_{i=1}^{m} \operatorname{deg} a_{p+i}(f)
$$

The proof of Proposition 3.3, and in particular Equation (1) since diam $E_{\Gamma}=0$ for the Nagao lattice $\Gamma$ as seen in Example 2.2, shows that the quadratic Lagrange spectrum with respect to the valuation at infinity of any quadratic irrational is contained in $\{0\} \cup\left\{q^{-n}\right.$ : $n \in \mathbb{N}\}$. The following result, which implies Theorem 1.1 (1) in the introduction, improves the upper bound of the spectrum. In Corollary 4.7 and Proposition 4.8, we will show that this upper bound is realised for certain quadratic irrationals.

Proposition 4.3. For every quadratic irrational $\alpha$, the spectrum $\operatorname{Sp}(\alpha)$ is contained in $\{0\} \cup\left\{q^{-n-2}: n \in \mathbb{N}\right\}$.

Proof. Up to replacing $\alpha$ by an element of $\Theta_{\alpha}$, we may assume that $\alpha \in \mathfrak{m}$ and $\alpha^{\sigma} \in \widehat{K}-\mathscr{O}$, so that the base point $*$ belongs to the geodesic line $] \alpha, \alpha^{\sigma}[$.

Let $f \in \widehat{K}-\left(K \cup \Theta_{\alpha}\right)$. Since $f$ is irrational, no positive subray of the geodesic line ]$\infty, f$ [ (oriented from $\infty$ to $f$ ) is contained in a single horoball of the canonical family $\left(\mathscr{H}_{\xi}\right)_{\xi \in \mathbb{P}^{1}(K)}$. Hence there exists a sequence $\left(x_{n}=\gamma_{n} *\right)_{n \in \mathbb{N}}$, with $\gamma_{n} \in \Gamma$, of vertices in the $\Gamma$-orbit of the base point $*$, converging to $f$ on the geodesic line $] \infty, f[$. As $*$ belongs to ]$\alpha, \alpha^{\sigma}$, we thus have that $] \infty, f[$ and $] \gamma_{n} \cdot \alpha, \gamma_{n} \cdot \alpha^{\sigma}\left[\right.$ meet at least at the vertex $\gamma_{n} *$.

The stabiliser $\mathrm{PGL}_{2}\left(\mathbb{F}_{q}\right)$ of $*$ in $\Gamma$ acts transitively on the set of pairs of distinct elements of the link of $*$. Thus, up to multiplying $\gamma_{n}$ on the right by an element of $\mathrm{PGL}_{2}\left(\mathbb{F}_{q}\right)$, the geodesic line ] $\infty, f[$ meets $] \gamma_{n} \cdot \alpha, \gamma_{n} \cdot \alpha^{\sigma}$ [ in a segment of length at least 2 for all $n \in \mathbb{N}$. Thus, by Lemma 3.1 (2), we have

$$
\min \left\{c\left(x, \gamma_{n} \cdot \alpha\right), c\left(x, \gamma_{n} \cdot \alpha^{\sigma}\right)\right\} \leq q^{-2} .
$$

Since $\left|\gamma_{n} \cdot \alpha-\gamma_{n} \cdot \alpha^{\sigma}\right|$ tends to 0 by Lemma 2.3, we have

$$
c_{\alpha}(f)=\liminf _{\beta \in \Theta_{\alpha},\left|\beta^{\sigma}-\beta\right| \rightarrow 0} c(x, \beta) \leq q^{-2} .
$$

Hence the result follows.

We are now going to give a series of computations of quadratic approximation constants. We start by two preliminary results.

Lemma 4.4. Let $\alpha \in \mathrm{QI}$ and let $f \in \widehat{K}-\left(K \cup \Theta_{\alpha}\right)$.

(1) If $m(f)>M(\alpha)$, then $c_{\alpha}(f) \geq q^{-M_{2}(\alpha)}$.

(2) If $M(f)<m(\alpha)$, then $c_{\alpha}(f) \geq q^{-M_{2}(f)}$.

Proof. (1) By the penetration properties of geodesic lines in the horoballs of the canonical family $\left(\mathscr{H}_{\xi}\right)_{\xi \in \mathbb{P}^{1}(K)}$, for every $\beta \in \Theta_{\alpha}$, the maximum height the geodesic line $] \beta^{\sigma}, \beta[$ enters in one of these horoballs is $M(\alpha)$. Similarly, the minimum height the geodesic line $] \infty, f[$ enters one of these horoballs except finitely many of them is $m(f)$, which is strictly bigger 
than $M(\alpha)$. Hence for all $\beta \in \Theta_{\alpha}$, the geodesic lines $] \infty, f[$ and $] \beta^{\sigma}, \beta[$ can meet at most in two consecutive horoballs $H_{n}(f)$ for $n \in \mathbb{N}$ large enough, and their intersection has length at most $M_{2}(\alpha)$ (and even at most $M(\alpha) \leq M_{2}(\alpha)$ if $] \beta^{\sigma}, \beta[$ meets at most one of the horoballs $H_{n}(f)$ for $n \in \mathbb{N}$ large enough), see the picture below.
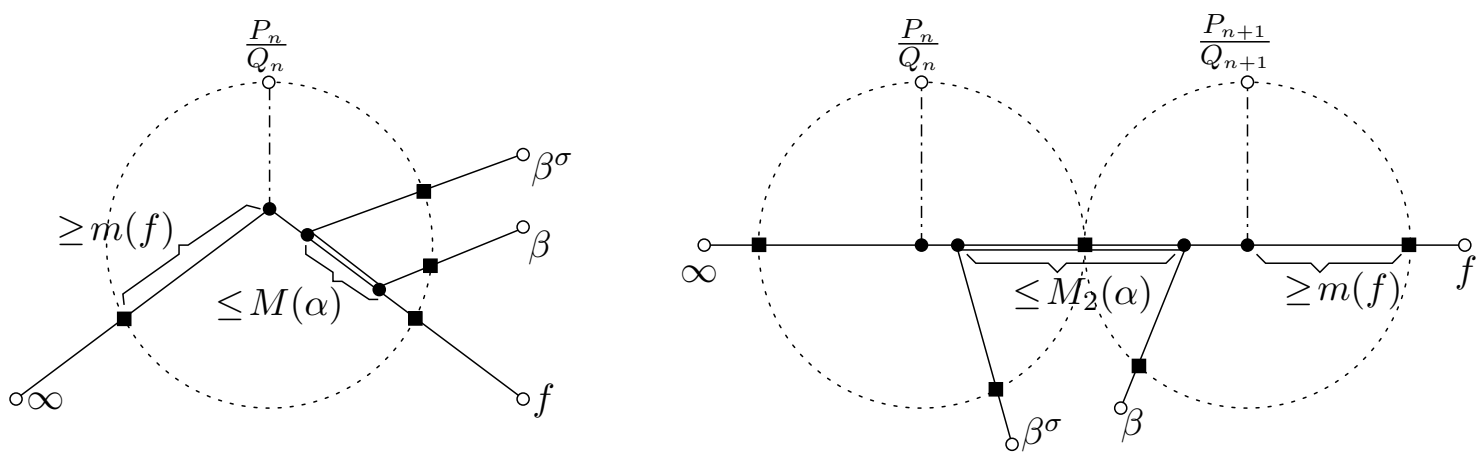

By Lemma 3.1, we have $c(f, \beta) \geq q^{-M_{2}(\alpha)}$ for all $\beta \in \Theta_{\alpha}$, which proves the result.

The proof of Assertion (2) is similar.

Lemma 4.5. For all $\alpha \in \mathrm{QI}$ and $f \in \widehat{K}-\left(K \cup \Theta_{\alpha}\right)$ such that $m(f)>M(\alpha)$, there exists a sequence $\left(\beta_{n}\right)_{n \in \mathbb{N}}$ in $\Theta_{\alpha}$ such that $c\left(f, \beta_{n}\right)=q^{-M_{2}(\alpha)}$ and $\left|\beta_{n}-\beta_{n}^{\sigma}\right| \rightarrow 0$ as $n \rightarrow+\infty$.

Proof. Replacing $\alpha$ by an element in its $\Gamma$-orbit if necessary, we can assume that the continued fraction expansion of $\alpha$ is periodic, that $\alpha \in \mathfrak{m}$ and $\alpha^{\sigma} \in \widehat{K}-\mathscr{O}$, and that $M_{2}(\alpha)=\operatorname{deg} a_{1}(\alpha)+\operatorname{deg} a_{2}(\alpha)$. The unipotent upper triangular subgroup $\left\{\left(\begin{array}{ll}1 & x \\ 0 & 1\end{array}\right)\right.$ : $x \in R\}$ of $\Gamma$ fixes $\infty \in \partial_{\infty} \mathbb{T}$, and acts transitively on the subset $R$ of $\partial_{\infty} \mathbb{T}$. Since the horoballs in the canonical family $\left(\mathscr{H}_{\xi}\right)_{\xi \in \mathbb{P}^{1}(K)}$ whose closure meets the closure of $\mathscr{H}_{\infty}$ are (besides $\mathscr{H}_{\infty}$ itself) the ones centred at an element of $R$, the group $\Gamma$ acts transitively on the ordered pairs of horoballs in this family whose closures meet at one point. In particular, for all $n \in \mathbb{N}$ large enough, there exists $\gamma_{n} \in \Gamma$ sending $H_{1}(\alpha)$ to $H_{n}(f)$ and $H_{2}(\alpha)$ to $H_{n+1}(f)$.

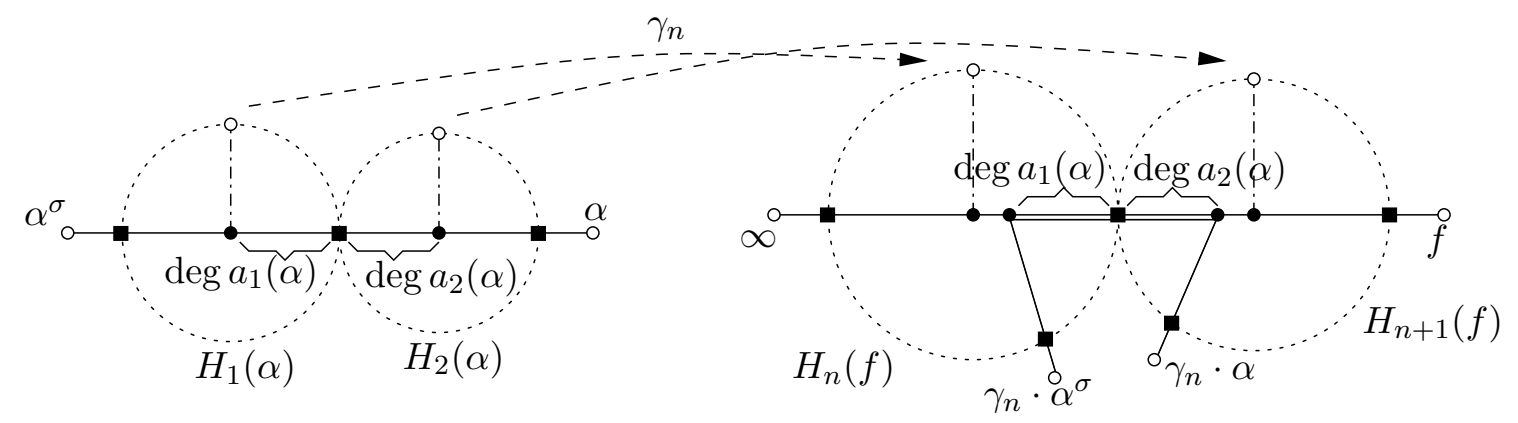

For all $x \in \mathbb{T}$ and $\gamma \in \operatorname{Aut}(\mathbb{T})$, and for every horoball $\mathscr{H}$ in $\mathbb{T}$, the height of $\gamma x$ with respect to $\gamma \mathscr{H}$ is equal to the height of $x$ with respect to $\mathscr{H}$. Hence for $n$ large enough, since $m(f)>M(\alpha)$, the geodesic lines $] \infty, f\left[\right.$ and $\left.\gamma_{n}\right] \alpha^{\sigma}, \alpha[=] \gamma_{n} \cdot \alpha^{\sigma}, \gamma_{n} \cdot \alpha[$ intersect along a segment of length equal to $\operatorname{deg} a_{1}(\alpha)+\operatorname{deg} a_{2}(\alpha)=M_{2}(\alpha)$. Let $\beta_{n}=\gamma_{n} \cdot \alpha$, we then have $c\left(f, \beta_{n}\right)=q^{-M_{2}(\alpha)}$ by Lemma 3.1. The fact that $\left|\beta_{n}-\beta_{n}^{\sigma}\right| \rightarrow 0$ as $n \rightarrow+\infty$ follows from Lemma 2.3. 
Corollary 4.6. Let $\alpha \in$ QI. If $f \in \widehat{K}-\left(K \cup \Theta_{\alpha}\right)$ satisfies $m(f)>M(\alpha)$, then $c_{\alpha}(f)=$ $q^{-M_{2}(\alpha)} \in \operatorname{Sp}(\alpha)$.

Proof. This follows immediately from Lemmas 4.4 (1) and 4.5, by the definition of the quadratic approximation constants.

Corollary 4.7. Let $\alpha \in$ QI. Then

(1) $\max \operatorname{Sp}(\alpha) \geq q^{-M_{2}(\alpha)}$.

(2) If $M(\alpha)=1$ or $m(\alpha) \geq 2$, then $\max \operatorname{Sp}(\alpha)=\max _{P \in \mathbb{F}_{q}[X], \operatorname{deg} P=1} c_{\alpha}([\bar{P}])=q^{-2}$.

Proof. (1) This follows from Corollary 4.6 since for instance

$$
f=\left[\overline{a_{p+1}(\alpha)+Y^{M(\alpha)+1}, a_{p}(\alpha)+Y^{M(\alpha)+1}}\right]
$$

satisfies the assumption of Corollary 4.6 if $p \in \mathbb{N}$ is such that $M_{2}(\alpha)=\operatorname{deg} a_{p}(\alpha)+$ $\operatorname{deg} a_{p+1}(\alpha)$.

(2) If $M(\alpha)=1$, then $M_{2}(\alpha)=2$ and this follows from Proposition 4.3 and Corollary 4.6. If $m(\alpha) \geq 2$, with $f=[\bar{Y}]$, we have $M(f)=1<m(\alpha)$ and $M_{2}(f)=2$, thus $c_{\alpha}(f) \geq q^{-2}$ by Lemma $4.4(2)$, and the result follows from Proposition 4.3.

The above corollary shows that the maximum Hurwitz constant is attained for many quadratic irrationals $\alpha$. In fact, the same holds for all quadratic irrationals with small enough period length.

Proposition 4.8. If $\alpha$ is a quadratic irrational over $K$ in $\widehat{K}$ whose period of its continued fraction expansion contains at most $q-2$ coefficients of degree 1 , then $\max \operatorname{Sp}(\alpha)=$ $\max _{P \in \mathbb{F}_{q}[X], \operatorname{deg} P=1} c_{\alpha}([\bar{P}])=q^{-2}$.

The first equality is a (strengthened) nonarchimedean version of the 2-periodic case of Bugeaud's conjecture solved by Lin [Lin, Rem. 1.3]. In particular, if $\alpha \in$ QI is eventually $k$-periodic with $k \leq q-1$ (as for instance with $k=2$ since $q \geq 3$ ), then $\max \operatorname{Sp}(\alpha)=\max _{P \in \mathbb{F}_{q}[X], \operatorname{deg} P=1} c_{\alpha}([\bar{P}])=q^{-2}$. Indeed, either all coefficients of the period of $\alpha$ have degrees 1 , in which case $M(\alpha)=1$ and Corollary 4.7 (2) applies, or $\alpha$ satisfies the assumption of Proposition 4.8. This proves Theorem 1.2 in the introduction.

Proof. Since $\operatorname{Card}\left(\mathbb{F}_{q}-\{0\}\right)=q-1$ and by the assumption, there exists a polynomial $P \in R$ of degree 1 such that for every degree 1 coefficient $a_{i}(\alpha)$ of the period of $\alpha$, the polynomial $P-a_{i}(\alpha)$ is nonconstant. Let $f=[\bar{P}]$. For all $\beta \in \Theta_{\alpha}$ and $n \in \mathbb{N}$ large enough, we claim that $] \beta^{\sigma}, \beta[$ agrees with $] \infty, f[$ on a segment with length at most 1 inside any horoball $H_{n}(f)$ for $n \in \mathbb{N}$. By an argument as in the proof of Proposition 4.3, this implies that $c_{\alpha}(f)=q^{-2}$. This in turn implies that $\max \operatorname{Sp}(\alpha) \geq q^{-2}$, and the result follows since $q^{-2}$ is an upper bound on $\max \operatorname{Sp}(\alpha)$ (see Proposition 4.3).

Assume for a contradiction that the geodesic segment $] \beta^{\sigma}, \beta[$ agrees with $] \infty, f[$ on a segment of length at least 2 inside $H_{n}(f)$. Since $\operatorname{deg} a_{n}(f)=\operatorname{deg} P=1$, this implies that $] \beta^{\sigma}, \beta$ [ and $] \infty, f$ [ actually coincide inside of $H_{n}(f)$. Assume that the orientations of the geodesic lines $] \beta^{\sigma}, \beta[$ and $] \infty, f$ [ respectively from $\beta^{\sigma}$ to $\beta$ and from $\infty$ to $f$ agree. By Lemma 4.1, this implies that if $a_{i}(\beta)$ is the coefficient in the period of $\beta$ such that $H_{n}(f)=H_{i}(\beta)$, then the polynomial $P-a_{i}(\beta)$ is constant. This implies that $\operatorname{deg} a_{i}(\beta)=1$ and this contradicts the definition of $P$, since $\alpha$ and $\beta$ have the same period (up to a cyclic permutation). 
If the period of a quadratic irrational $\alpha$ is longer than $q-1$, then its Hurwitz constant $\max \operatorname{Sp}(\alpha)$ may be arbitrarily small, as the following result shows.

Proposition 4.9. For all $m, k \in \mathbb{N}-\{0,1\}$, let us denote by $\left\{b_{1}, \ldots, b_{N}\right\}$ the set $t^{5}$ of the finite sequences of length $m$ of polynomials of degree at least 1 and at most $k$, and let

$$
\mathscr{E}_{m, k}=\bigcup_{\sigma \in \mathscr{S}_{N}} \Gamma \cdot\left[\overline{b_{\sigma(1)}, \ldots, b_{\sigma(N)}}\right]
$$

For every $\alpha \in \mathscr{E}_{m, k}$, we have $\max \operatorname{Sp}(\alpha) \leq q^{-\min (2 m, k+2)}$.

Proof. Let us fix $\alpha \in \mathscr{E}_{m, k}$ and $f \in \widehat{K}-\left(K \cup \Theta_{\alpha}\right)$, and let us prove that $c_{\alpha}(f) \leq$ $q^{-\min (2 m, k+2)}$, which gives the result.

Assume first that there exists a subsequence of coefficients $\left(a_{i_{n}}(f)\right)_{n \in \mathbb{N}}$ of $f$ such that $i_{0} \geq 1$ and $\operatorname{deg} a_{i_{n}}(f)>k$ for all $n \in \mathbb{N}$. Let $x_{n}$ be the point of $\partial H_{i_{n}}(f)$ at which the geodesic line ] $\infty, f$ [ exits the horoball $H_{i_{n}}(f)$. Since any polynomial $P \in R$ of degree $k$ occurs as a coefficient of $\alpha$ (in its periodic part), there exists a horoball $\mathscr{H}_{k}$ in the canonical family $\left(\mathscr{H}_{\xi}\right)_{\xi \in \mathbb{P}^{1}(K)}$ which intersects the geodesic $] \alpha^{\sigma}, \alpha[$ in a segment of length exactly $2 k$. Let $y$ be the point of $\partial \mathscr{H}_{k}$ at which the geodesic $] \alpha^{\sigma}, \alpha\left[\right.$ exits the horoball $\mathscr{H}_{k}$.

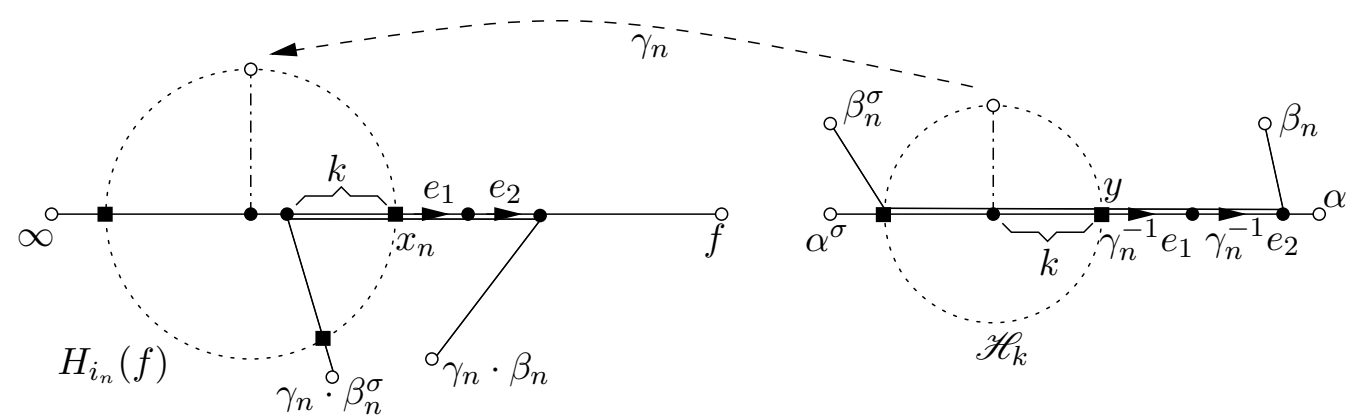

By the transitivity of the action of $\Gamma$ on the pairs $(\mathscr{H}, x)$ where $\mathscr{H}$ belongs to the canonical family $\left(\mathscr{H}_{\xi}\right)_{\xi \in \mathbb{P}^{1}(K)}$ and $x$ belongs to $\partial \mathscr{H}$, there exists $\gamma_{n} \in \Gamma$ which sends $\left(\mathscr{H}_{k}, y\right)$ to $\left(H_{i_{n}}(f), x_{n}\right)$. Let $e_{1}, e_{2}$ be the consecutive edges along ] $\infty, f$ [ such that the origin of $e_{1}$ is $x_{n}$. Note that the endpoint of $e_{2}$ (which is at distance 2 from $x_{n}$ ) might belong to the orbit $\Gamma *$ of the base point of $\mathbb{T}$ or not. Since any pair $\left(P, P^{\prime}\right)$ with $P, P^{\prime} \in R, \operatorname{deg} P=k$ and $\operatorname{deg} P^{\prime} \in\{1,2\}$ occurs as a pair of consecutive coefficients in the continued fraction expansion of $\alpha$, there exists $\beta_{n} \in \Theta_{\alpha}$ such that the geodesic line ] $\beta_{n}^{\sigma}, \beta_{n}$ [ contains $\gamma_{n}^{-1} e_{1}$ and $\gamma_{n}^{-1} e_{2}$, and coincides with $] \alpha^{\sigma}, \alpha$ [inside $\mathscr{H}_{k}$. Hence $\left.\gamma_{n}\right] \beta_{n}^{\sigma}, \beta_{n}$ [ coincides with ] $\infty, f[$ on $e_{1}, e_{2}$ as well as on a segment of length exactly $k$ inside $H_{i_{n}}(f)\left(\right.$ since $\operatorname{deg} a_{i_{n}}(f)>k$ ). Thus

$$
\min \left\{c\left(f, \gamma_{n} \cdot \beta_{n}^{\sigma}\right), c\left(f, \gamma_{n} \cdot \beta_{n}\right)\right\} \leq q^{-(k+2)}
$$

by Lemma 3.1. Since $\left|\gamma_{n} \cdot \beta_{n}-\gamma_{n} \cdot \beta_{n}^{\sigma}\right| \rightarrow 0$ as $n \rightarrow+\infty$ by Lemma 2.3, we have $c_{\alpha}(f) \leq q^{-(k+2)}$.

Otherwise, there exists $i_{0} \in \mathbb{N}$ such that $\operatorname{deg} a_{i}(f) \leq k$ for all $i \geq i_{0}$. For every $n \in \mathbb{N}$, consider the string of $m$ consecutive horoballs $H_{i_{0}+n m}(f), \ldots, H_{i_{0}+(n+1) m-1}(f)$ crossed by the geodesic line $] \infty, f[$. The quadratic irrational $\alpha$ has been constructed in such a way that there exists a string of consecutive coefficients in the period of $\alpha$ that agrees with those

\footnotetext{
${ }^{5}$ with an arbitrary ordering and $N=\left(q^{k+1}-q\right)^{m}$
} 
of $f$ inside the above horoballs. Using Lemma 4.2, this gives the estimate $c_{\alpha}(f) \leq q^{-2 m}$. Together with the previous estimate, this completes the proof.

Example 4.10. If $\alpha$ belongs to the set $\mathscr{E}_{m, k}$ constructed in Proposition 4.9 with $k=m=2$, then

$$
\max \operatorname{Sp}(\alpha)=\max _{P \in R} c_{\alpha}([\bar{P}])=\limsup _{P \in R, \operatorname{deg} P \rightarrow+\infty} c_{\alpha}([\bar{P}])=q^{-4} .
$$

Indeed, Proposition 4.9 gives $\max \operatorname{Sp}(\alpha) \leq q^{-4}$. For any $P \in R$ with $\operatorname{deg} P \geq 3$ and $f=[\bar{P}]$, we have $c_{\alpha}(f)=q^{-4}$ by Corollary 4.6. The result follows.

Let

$$
\varphi=[\bar{Y}] .
$$

As a special case of Corollary 4.7, we get $\max \operatorname{Sp}(\varphi)=q^{-2}$. The following result gives in particular a complete description of the quadratic Lagrange spectrum of $\varphi$, proving Theorem 1.3 in the introduction.

Theorem 4.11. For every $P \in R$ with $\operatorname{deg} P=1$, we have

$$
\operatorname{Sp}([\bar{P}])=\{0\} \cup\left\{q^{-(2+n)}: n \in \mathbb{N}\right\} .
$$

Proof. Let $\alpha=[\bar{P}]$. For every $m \in \mathbb{N}$, let $f_{m}=\left[0, \overline{Y^{2}, P, P, \ldots, P}\right]$ and $f_{m}^{\prime}=$ $\left[0, \overline{Y^{2}, P, P, \ldots, P, P+1}\right]$, which are elements of $K^{(2)}-\Theta_{\alpha}$ with period lengths of the periodic part of their continued fraction equal to $m+1$ and $m+2$ respectively. Let us prove that $c_{\alpha}\left(f_{m}\right)=q^{-2 m-2}$ and $c_{\alpha}\left(f_{m}^{\prime}\right)=q^{-2 m-3}$, which implies that $q^{-n-2}$ belongs to $\operatorname{Sp}(\alpha)$ for all $n \in \mathbb{N}$. This gives the result by Proposition 4.3.

We know that $c_{\alpha}\left(f_{0}\right)=q^{-2}$ by Corollary 4.6. Assume that $m \geq 1$. Note that for every $n \in \mathbb{N}$, the maximum height of the geodesic line ] $\infty, f_{m}$ [ inside the horoball $H_{n(m+1)+i+1}\left(f_{m}\right)$ is equal to 2 if $i=0, m+1$ and to 1 if $1 \leq i \leq m$. Since the geodesic lines $] f_{m}^{\sigma}, f_{m}$ [ and $] \alpha^{\sigma}, \alpha$ [ both contain points in $\Gamma *$, some image of $] \alpha^{\sigma}, \alpha$ [ by an element of $\Gamma$ meets $] f_{m}^{\sigma}, f_{m}\left[\right.$. Hence by Remark 3.2, we have $c_{\alpha}\left(f_{m}\right)=q^{-n\left(f_{m}, \alpha\right)}$ where

$$
n\left(f_{m}, \alpha\right)=\max _{\gamma \in \Gamma} \operatorname{length}(] f_{m}^{\sigma}, f_{m}[\cap \gamma] \alpha^{\sigma}, \alpha[) .
$$

By Lemma 4.2, for every $n \in \mathbb{N}$, there exists $\beta_{n} \in \Theta_{\alpha}$ such that the geodesic line $] \beta_{n}^{\sigma}, \beta_{n}[$ coincides with the geodesic line ] $\infty, f_{m}$ [ inside the horoballs $H_{n(m+1)+i+1}\left(f_{m}\right)$ for $1 \leq i \leq$ $m$. Therefore $n\left(f_{m}, \alpha\right) \geq 2 m$. For every such $\beta_{n}$, the height of the geodesic line $] \beta_{n}^{\sigma}, \beta_{n}$ [ inside $H_{n(m+1)+i+1}\left(f_{m}\right)$ for $i=0, m+1$ is at most 1 . Hence for $n$ large enough, the geodesic lines ] $\infty, f_{m}[$ and $] \beta_{n}^{\sigma}, \beta_{n}$ [ coincide at most in one edge inside $H_{n(m+1)+i+1}\left(f_{m}\right)$ for $i=0, m+1$. Thus $n\left(f_{m}, \alpha\right) \leq 2 m+2$. Since the stabiliser in $\Gamma$ of both $*$ and the edge $e_{\infty}$ of $\operatorname{lk}(*)$ pointing towards $\infty$ acts transitively on the edges of $\operatorname{lk}(*)$ different from $e_{\infty}$, it is possible to adjust $\beta_{n}$ so that ] $\infty, f_{m}$ [ and $] \beta_{n}^{\sigma}, \beta_{n}$ [ do coincide in exactly an edge inside $H_{n(m+1)+i+1}\left(f_{m}\right)$ for both $i=0, m+1$. Thus $n\left(f_{m}, \alpha\right)=2 m+2$, as wanted.

Similarly, for every $n \in \mathbb{N}$, consider the elements $\beta_{n}^{\prime} \in \Theta_{\alpha}$ such that the geodesic line ]$\beta_{n}^{\prime \sigma}, \beta_{n}^{\prime}$ [ coincides with the geodesic line ] $\infty, f_{m}^{\prime}$ [ inside the horoballs $H_{n(m+2)+i+1}\left(f_{m}^{\prime}\right)$ for $1 \leq i \leq m$. Using the fact that the upper unipotent action of $\mathbb{F}_{q}[X]$ is simply transitive on the edges going out of the horoball $\mathscr{H}_{\infty}$, and that two polynomials have the same action on $\partial \mathscr{H}_{\infty}$ if and only if they differ by a constant, it is possible to adjust $\beta_{n}^{\prime}$ so that for $n$ large enough $] \infty, f_{m}^{\prime}[$ and $] \beta_{n}^{\prime \sigma}, \beta_{n}^{\prime}$ [ do coincide in exactly one edge inside $H_{n(m+2)+1}\left(f_{m}^{\prime}\right)$ and two 
edges inside $H_{n(m+2)+m+1}\left(f_{m}^{\prime}\right)$, but do not coincide in an edge inside $H_{n(m+2)+m+2}\left(f_{m}^{\prime}\right)$. Thus $n\left(f_{m}^{\prime}, \alpha\right)=2 m+3$, as wanted.

The next result shows that there are examples of quadratic Lagrange spectra which contain a gap, that is, are not always of the form $\{0\} \cup\left\{q^{-n}: n \in \mathbb{N}, n \geq N\right\}$ for some $N \in \mathbb{N}$

Proposition 4.12. Let $k \in \mathbb{N}-\{0,1\}$, let $\left\{b_{1}, \ldots, b_{N^{\prime}}\right\}$ be the set $t^{6}$ of the polynomials of degree at least $k$ and at most $2 k$, and let $\mathscr{F}_{k}=\bigcup_{\sigma \in \mathscr{S}_{N^{\prime}}} \Gamma \cdot\left[\overline{b_{\sigma(1)}, \ldots, b_{\sigma\left(N^{\prime}\right)}}\right]$. For every $\alpha \in \mathscr{F}_{k}$, the number $q^{-2 k+1}$ does not belong to $\operatorname{Sp}(\alpha)$.

Proof. Let $f \in \widehat{K}-\left(K \cup \Theta_{\alpha}\right)$. Let us prove that $c_{\alpha}(f) \neq q^{-2 k+1}$, which gives the result. There are three cases to consider.

Assume first that there exists $i_{0} \in \mathbb{N}$ such that $\operatorname{deg} a_{i}(f)<k$ for all $i \geq i_{0}$. By Lemma 4.4 (2), we have $c_{\alpha}(f) \geq q^{-M_{2}(f)} \geq q^{-2(k-1)}=q^{-2 k+2}$, and in particular $c_{\alpha}(f) \neq q^{-2 k+1}$.

Assume then that there exists a subsequence of coefficients $a_{i_{n}}(f)$ for $n \in \mathbb{N}$ such that $i_{0} \geq 1$ and $k \leq \operatorname{deg} a_{i_{n}}(f) \leq 2 k$. Then $a_{i_{n}}(f) \in\left\{b_{1}, \ldots, b_{N^{\prime}}\right\}$, and again there exists an element $\beta_{i_{n}} \in \Theta_{\alpha}$ for which the intersection $] \beta_{i_{n}}^{\sigma}, \beta_{i_{n}}[\cap] \infty, f$ [ has length at least $2 \operatorname{deg} a_{i_{n}}(f) \geq 2 k$. Hence $c_{\alpha}(f) \leq q^{-2 k}$, and in particular $c_{\alpha}(f) \neq q^{-2 k+1}$.

If neither of the previous two cases occurs, we have $\operatorname{deg} a_{i}(f)>2 k$ for $i$ large enough. By Lemma 4.5, we have $c_{\alpha}(f) \leq q^{-4 k}$, and in particular $c_{\alpha}(f) \neq q^{-2 k+1}$.

Corollary 4.13. Let $\alpha$ be as in Proposition 4.12. Then $\operatorname{Sp}(\alpha)$ contains a gap.

Proof. By Corollary 4.7, we have $\max \operatorname{Sp}(\alpha)=q^{-2}$, and $\operatorname{Sp}(\alpha)$ contains $q^{-n}$ for all $n$ large enough by Theorem 3.4. Thus, the spectrum has a gap that contains $q^{-2 k+1}$ by Proposition 4.12 .

\section{References}

[BaL] H. Bass and A. Lubotzky. Tree lattices. Prog. in Math. 176, Birkhäuser, 2001.

[BeN] V. Berthé and H. Nakada. On continued fraction expansions in positive characteristic: equivalence relations and some metric properties. Expo. Math. 18 (2000) 257-284.

[BH] M. R. Bridson and A. Haefliger. Metric spaces of non-positive curvature. Grund. math. Wiss. 319, Springer Verlag, 1999.

[BPP] A. Broise-Alamichel, J. Parkkonen, and F. Paulin. Equidistribution and counting under equilibrium states in in negative curvature and trees. Applications to non-Archimedean Diophantine approximation. Book preprint (365 pages) arXiv:1612.06717, with an Appendix by J. Buzzi, to appear in Progress in Mathematics.

[Bug1] Y. Bugeaud. On the quadratic Lagrange spectrum. Math. Z. 276 (2014) 985-999.

[Bug2] Y. Bugeaud. Nonarchimedean quadratic Lagrange spectra and continued fractions in power series fields. Preprint arXiv:1804.03566.

[CF] T. Cusick and M. Flahive. The Markoff and Lagrange spectra. Math. Surv. Mono. 30, Amer. Math. Soc. 1989.

[Gos] D. Goss. Basic structures of function field arithmetic. Erg. Math. Grenz. 35, Springer Verlag 1996.

\footnotetext{
${ }^{6}$ with an arbitrary order and $N^{\prime}=q^{2 k+1}-q^{k}$
} 
[HP] S. Hersonsky and F. Paulin. On the almost sure spiraling of geodesics in negatively curved manifolds. J. Diff. Geom. 85 (2010) 271-314.

[Khi] A. Y. Khinchin. Continued fractions. Univ. Chicago Press, 1964.

[Las] A. Lasjaunias. A survey of Diophantine approximation in fields of power series. Monat. Math. 130 (2000) 211-229.

[Lin] X. Lin. Quadratic Lagrange spectrum: I. Math. Z. 289 (2018) 515-533.

[PaP1] J. Parkkonen and F. Paulin. Sur les rayons de Hall en approximation diophantienne. Comptes Rendus Math. 344 (2007) 611-614.

[PaP2] J. Parkkonen and F. Paulin. On the closedness of approximation spectra. J. Th. Nb. Bordeaux 21 (2009) 701-710.

[PaP3] J. Parkkonen and F. Paulin. Prescribing the behaviour of geodesics in negative curvature. Geom. \& Topo. 14 (2010) 277-392.

[PaP4] J. Parkkonen and F. Paulin. Spiraling spectra of geodesic lines in negatively curved manifolds. Math. Z. 268 (2011) 101-142, Erratum: Math. Z. 276 (2014) 1215-1216.

[Pau] F. Paulin. Groupe modulaire, fractions continues et approximation diophantienne en caractéristique p. Geom. Dedi. 95 (2002) 65-85.

[Pej] T. Pejković. Quadratic Lagrange spectrum. Math. Z. 283 (2016) 861-869.

[Poi] G. Poitou. Sur l'approximation des nombres complexes par les nombres des corps imaginaires quadratiques dénués d'idéaux non principaux particulièrement lorsque vaut l'algorithme d'Euclide. Ann. Scien. Ec. Norm. Sup. 70 (1953) 199-265.

[Ros] M. Rosen. Number theory in function fields. Grad. Texts Math. 210, Springer-Verlag, 2002 .

[Sch1] A. L. Schmidt. Farey simplices in the space of quaternions. Math. Scand. 24 (1969) 31-65.

[Sch2] A. L. Schmidt. Diophantine approximation of complex numbers. Acta Math. 134 (1975) $1-85$.

[Sch] W. Schmidt. On continued fractions and diophantine approximation in power series fields. Acta Arith. XCV (2000) 139-166.

[Ser] J.-P. Serre. Arbres, amalgames, $S L_{2}$. 3ème éd. corr., Astérisque 46, Soc. Math. France, 1983.

Department of Mathematics and Statistics, P.O. Box 35

40014 University of Jyväskylä, FINLAND.

e-mail: jouni.t.parkkonen@jyu.fi

Laboratoire de mathématique d'Orsay, UMR 8628 Univ. Paris-Sud et CNRS, Université Paris-Saclay,

91405 ORSAY Cedex, FRANCE

e-mail: frederic.paulin@math.u-psud.fr 